\begin{tabular}{|c|c|c|}
\hline $\begin{array}{l}\text { ITC 2/47 } \\
\text { Journal of Information Technol }\end{array}$ & \multicolumn{2}{|c|}{$\begin{array}{c}\text { Leakage-Resilient Certificateless Signature Under } \\
\text { Continual Leakage Model }\end{array}$} \\
\hline $\begin{array}{l}\text { Vol. } 47 / \text { No. } 2 / 2018 \\
\text { pp. } 363-386\end{array}$ & Received 2017/09/12 & Accepted after revision 2018/04/15 \\
\hline $\begin{array}{l}\text { DOI 10.5755/j01.itc.47.2.17847 } \\
\text { ๑ Kaunas University of Technology }\end{array}$ & \multicolumn{2}{|c|}{ crossef http://dx.doi.org/10.5755/j01.itc.47.2.17847 } \\
\hline
\end{tabular}

\title{
Leakage-Resilient Certificateless Signature Under Continual Leakage Model
}

\author{
Jui-Di Wu, Yuh-Min Tseng and Sen-Shan Huang \\ Department of Mathematics, National Changhua University of Education, Changhua 500, Taiwan
}

Corresponding author:ymtseng@cc.ncue.edu.tw

In the past, the security notions of cryptography were modeled under the assumption that private (or secret) keys are completely hidden to adversaries. Nowadays, these security notions could be insufficient due to a new kind of threat, called "side-channel attacks", by which an adversary obtains partial information of private (or secret) keys via employing specific properties resulting from physical implementations of cryptographic schemes. In order to resist such side-channel attacks, numerous leakage-resilient cryptographic schemes have been proposed. However, there is little work on studying leakage-resilient certificateless cryptographic schemes. In this article, we propose the first leakage-resilient certificateless signature (LR-CLS) scheme under the continual leakage model. In the generic bilinear group model, we demonstrate that our scheme possesses existential unforgeability against adaptive chosen-message attacks for both Type I and Type II adversaries. Finally, performance analysis is made to demonstrate that the proposed LR-CLS scheme is suitable for resource-constrained devices.

KEYWORDS: Side-channel attack, Certificateless signature, Leakage-resilience, Provable security.

\section{Introduction}

In the conventional public key settings [14,33], a certificate is employed to validate the mapping between a user's identity and her/his associated public key. In order to remove the certificate usage, Shamir [36] introduced the concept of identity (ID)-based public key setting. Based on Shamir's concept, Boneh and Franklin [7] proposed the first practical construction of ID- based encryption (IBE) from bilinear pairings. In an ID-based public key setting, identity information of a user is viewed as the user's public key, by which a trusted private key generator (PKG) can produce and send the corresponding private key to the user. Under this circumstance, the PKG knows private keys of all the users. In other words, all the ID-based public key 
settings suffer from the key escrow problem in the sense that the PKG may decrypt all the ciphertexts or sign the messages on behalf of all users.

In 2003, Al-Riyami and Paterson [1] proposed a new public key paradigm, termed certificateless public key setting (CL-PKS), to resolve the key escrow problem mentioned above. In CL-PKS, a user's private key consists of two components, namely, an initial key and a secret key. In addition, there exists a semi-trusted third party, called the key generation center (KGC), who is responsible to produce the user's initial key by its system secret key and the user's identity information. Meanwhile, the user randomly chooses a secret key and computes the corresponding public key without requiring any certificate. Hence, the KGC cannot access the user's private key due to the lack of the secret key generated by the user. Therefore, the CL-PKS not only resolves the key escrow problem in ID-based public key settings but also removes the certificate management in conventional public key public key settings. In the past decade, the research on CL-PKS has great progress and numerous cryptographic schemes have been proposed [19-24, 29, 30, 39, 40, 44, 46].

The security notions for these public key settings mentioned above (including conventional, ID-based and certificateless) were modeled under the assumption that both the system's and users' private (or secret) keys are completely hidden to an adversary. Nowadays, these security notions could be insufficient due to a new kind of threat, called "side-channel attacks", such as fault attack [4, 6], power analysis [27], timing attack [10, 28], etc. For side-channel attacks, an adversary may obtain partial information of private (or secret) keys by employing specific properties resulting from physical implementations of cryptographic schemes. Thus, even if a cryptographic scheme was proven secure in an adversary model without addressing side-channel attacks, the cryptographic scheme could be broken in an environment where an adversary may obtain the partial information of private (or secret) keys. Leakage-resilient cryptography provides a solution to counteract side-channel attacks. Very recently, the study of leakage-resilient cryptography has received significant attention. Based on conventional public key settings, numerous leakage-resilient public key encryption schemes $[2,11,26,32]$ and leakage-resilient signature schemes [3, 15, 16, 18, 25, 38] have been proposed.

\subsection{Related Work}

The security notion of leakage-resilient cryptography is that a cryptographic scheme is still secure even if the partial leakage information of the private (or secret) keys involved in the scheme is visible to the adversary. In order to represent the leakage resilience of cryptographic schemes, adversary models must define the capabilities of an adversary leaking the partial information of the private (or secret) keys. For representing the leakage ability of an adversary, there are two kinds of leakage models, namely, bounded leakage model and continual leakage model, which are described as follows. Typically, a cryptographic scheme consists of several computation rounds. In leakage-resilient cryptography, a leakage function $f$ is given and $f(\tau)$ is viewed as the leakage information, where $\tau$ indicates the data (including permanent and temporary secret values) accessed during the current computation round. The outputlength of $f$ is restricted to $\lambda$ bits, that is, the leakage information of each computation round is bounded. On the other hand, if the total leakage information of a cryptographic scheme is unbounded, the whole private key would completely be revealed to the adversary so that it will injure the security of the cryptographic scheme. Hence, several leakage-resilient cryptographic schemes [3, 25] make a restriction on the overall leakage information to be bounded. This is called the bounded leakage model. However, this restriction is not practical. In recently proposed leakage-resilient cryptographic schemes, the continual leakage model is the most accredited model for leakage ability of an adversary, which provides the overall unbounded leakage property than the bounded leakage model. The continual leakage model possesses the following properties [9, 12, 18]:

Only computation leakage: Only temporary and permanent secret values currently accessed in a computation round could be leaked to a side-channel adversary.

_ Bounded leakage of single observation: The secret information leaked by single computation round (or called an observation) is bounded to $\lambda$ bits. This property bounds the leakage of each computation round to some fraction of secret information.

- Independent leakage: The leakage information of each computation round is independent of the other computation rounds. 
- Overall unbounded leakage: The overall amount of leakage information is assumed to be unbounded. Hence, after (or before) each computation round, the secret value must be refreshed (updated). It is obvious that the leakage bound can be restricted between any two successive secret value refreshes.

Based on conventional public key settings, several leakage-resilient public key encryption and signature schemes were proposed under the continual leakage model, which are surveyed as follows. In 2010, Kiltz and Pietrzak [26] proposed a leakage-resilient public key encryption in the generic bilinear group (GBG) model [5]. The GBG model is viewed as a kind of security proving technique, which will be defined in Section 2. It is worth mentioning, that the GBG model may be employed in the security proofs of cryptographic schemes under non-leakage model, bounded leakage model and continual leakage model. Following Kiltz and Pietrzak's technique in the GBG model, Galindo and Vivek [18] proposed a secure leakage-resilient signature scheme. Afterwards, based on Boneh et al.'s short signature [8] and GBG model, Tang et al. [38] presented an improved leakage-resilient signature scheme which reduces one exponential computation compared with Galindo and Vivek's scheme [18]. The security of Tang et al.'s scheme is based on both the GBG model and the random oracle model. In 2016, based on the generic bilinear group, Galindo et al. [17] also presented and implemented a new leakage-resilient ElGamal public key encryption scheme, which is the newest implementation for leakage-resilient protocols in the GBG model.

In ID-based public key settings, Brakerski et al. [9] proposed the first leakage-resilient ID-based encryption (LR-IBE) scheme under the continual leakage model. Afterwards, Yuen et al. [45] proposed an improved LR-IBE scheme to improve performance. In 2016, the first leakage-resilient ID-based signature (LR-IBS) was proposed by Wu et al. [42]. Under the continual leakage model, their LR-IBS scheme allows an adversary to learn partial information of both the system secret key in the key extract phase and the user's private key in the signing phase during the entire lifetime of the system. Also, their LR-IBS scheme possesses existential unforgeability against ID and adaptive chosen message (EUF-CMA) attacks. Nevertheless, Wu et al.'s LR-IBS scheme is constructed under the ID-based public key settings, so it suffers from the key escrow problem mentioned earlier.

\subsection{Contribution and Organization}

In the past, there is little work on studying the design of leakage-resilient certificateless cryptographic schemes. In 2013, Xiong et al. [43] proposed the first leakage-resilient certificateless public key encryption scheme (with various leakage conditions) for Type I adversary (outsider) and Type II adversary (honest-but-curious KGC), following the classification in traditional certificateless public key encryption [24]. However, Xiong et al.'s scheme did not resist adaptive chosen-ciphertext key-leakage attacks (INDKL-CCA2). In 2016, Zhou et al. [47] improved Xiong et al.'s scheme to propose an IND-KL-CCA2-secure certificateless signcryption scheme based on bilinear pairings. Both Xiong et al.'s and Zhou et al. schemes are secure under the bounded leakage model, but not under the continual leakage model.

Up to now, no work has been done on the design of leakage-resilient certificateless signature (LR-CLS). In this article, we will propose the first leakage-resilient certificateless signature scheme under the continual leakage model. We first define the security notions for LR-CLS schemes under the continual leakage model. The security notions include two kinds of attackers, namely, Type I adversary (outsider) and Type II adversary (honest-but-curious KGC). Both kinds of adversaries are extended from the security notions of traditional certificateless signature (CLS) schemes by adding the key leakage queries. Under the continual leakage model, the proposed LR-CLS scheme is allowed to leak partial information of the system secret key in the initial key extract phase and the user's private key in the signing phase. In the generic bilinear group model, we demonstrate that our scheme possesses existential unforgeability against adaptive chosen-message attacks for both Type I and Type II adversaries. Finally, performance analysis is made to demonstrate that the proposed LR-CLS scheme is suitable for resource-constrained devices.

The rest of the paper is organized as follows. In Section 2 , we present preliminaries. The framework and security notions of LR-CLS schemes are defined in Section 3, while a concrete LR-CLS scheme is proposed in Section 4. The security of the proposed LR-CLS scheme is formally proved in Section 5. In 
Section 6, we demonstrate the performance analysis of the proposed LR-CLS scheme. Conclusions are drawn in Section $\%$.

\section{Preliminaries}

In this section, we briefly introduce the concepts of bilinear groups [7, 35, 41], the notions of the generic bilinear group model $[5,18,42]$ and the entropy.

\subsection{Bilinear Groups}

Let $G$ denote a multiplicative group of large prime order $p$ while $G_{T}$ is also a multiplicative cyclic group with the same order. Assume that $g$ is an arbitrary generator of $G$. An admissible bilinear pairing is a map $e: G \times G \rightarrow G_{T}$ which satisfies the following three properties:

_ Bilinearity: $e\left(g_{1}^{a}, g_{2}^{b}\right)=e\left(g_{1}, g_{2}\right)^{a b}$, where $g_{1}, g_{2} \in G$ and $a, b \in Z_{p}^{*}$.

- Non-degeneracy: $e(g, g) \neq 1$, where $g \in G$.

_ Computability: $\left(g_{1}, g_{2}\right)$ can be efficiently computed, where $g_{1}, g_{2} \in G$.

Meanwhile, $G$ is called a bilinear group and $G_{T}$ is the target group of the admissible bilinear map $e$. A reader can refer to previous literatures such as [7, 35, 41] for a more comprehensive description of groups, maps and other parameters.

\subsection{Generic Bilinear Groups Model}

In 1997, Shoup [37] introduced the notions of the generic group model which is viewed as a kind of security proving technique for cryptographic schemes. In this model, the adversary is only given access to a randomly chosen encoding of a group controlled by a challenger. Basically, the model includes an oracle that executes the group operation [31] which takes as input two group elements $a$ and $b$, and outputs $a^{*} b$, where * denotes the group operation. One of the main usages of the generic group model is to analyze computational hardness assumption, i.e. the discrete logarithm problem in a group. If an adversary can efficiently find a collision encoding of a group operation, it is said to solve the computational hardness assumption.

Boneh et al. [5] presented the generic bilinear group (GBG) model, which is an extension of the generic group model. In the generic bilinear group model, there are two multiplicative groups $G$ and $G_{T}$. Both groups $G$ and $G_{T}$ have their own multiplication operation. Additionally, there exists a bilinear pairing operation to map two elements of $G$ to one element of $G_{T}$. Therefore, the elements of $G$ and $G_{T}$ are encoded by two random injective maps $\varepsilon: Z_{p} \rightarrow \Xi$ and $\varepsilon_{T}: Z_{p} \rightarrow \Xi_{T}$, respectively, where $\Xi$ and $\Xi_{T}$ are bit strings while $\Xi \cap \Xi_{T}=\phi$ and $|\Xi /=| \Xi_{T} \mid=p$. The operations in $G, G_{T}$ and the evaluation of the bilinear map $e$ are performed by three public oracles $O, O_{T}$ and $O_{P}$, respectively. For any $a, b \in Z_{p}^{*}$, we have the following properties:

_ $O(\varepsilon(a), \varepsilon(b)) \rightarrow \varepsilon(a+b \bmod p)$.

- $O_{T}\left(\varepsilon_{T}(a), \varepsilon_{T}(b)\right) \rightarrow \varepsilon_{T}(a+b \bmod p)$.

- $O_{P}(\varepsilon(a), \varepsilon(b)) \rightarrow \varepsilon_{T}(a b \bmod p)$.

Note that if $g$ is a generator of the group $G$, we have $g=\varepsilon(1)$ and $g_{T}=e(g, g)=\varepsilon_{T}(1)$.

\subsection{Entropy}

Entropy is a measure of the number of possible microscopic states (or microstates) of a system in thermodynamic equilibrium. The interpretation of entropy in statistical mechanics is the measure of uncertainty. Let $X$ be a finite random variable and $P r$ be the associated probability distribution. Min-entropy is a way of measuring the worst-case predictability of a random variable. We define two kinds of min-entropies as follows:

1 The min-entropy of a finite random variable $X$ is defined as $H_{\infty}(X)=-\log _{2}\left(\max _{x} \operatorname{Pr}[X=x)\right]$.

2 The average conditional min-entropy of $X$ under a given correlated random variable $Z$ is defined as $\widetilde{H}_{\infty}(X \mid Z)=-\log _{2}\left(E_{z \leftarrow Z}[\max \operatorname{Pr}[X=x \mid Z=z]]\right)$.

Dodis et al. [13] provided the following result on the entropy.

Lemma 1. Let $f: X \rightarrow\{0,1\}^{\prime \prime}$ be a leakage function on a given random variable $X$, where $\lambda^{\prime}$ is a fixed length. We have $\widetilde{H}_{\infty}\left(X \mid f(X) \geqq H_{\infty}(X)-\lambda^{\prime}\right.$.

Furthermore, Galindo and Vivek [18] presented a result (Lemma 2 below) to measure the probability distribution of polynomial under the advantage of leakage information, which is a variant of the Schwartz-Zippel lemma [34, 48]. Based on Lemma 2, a direct result (Corollary 1 below) is obtained.

Lemma 2. Let $F \in Z_{p}\left[X_{1}, X_{2}, \ldots, X_{n}\right]$ be a non-zero polynomial of total degree at most $d$. Let $P_{i}$ (for $i=1,2, \ldots$, $n$ ) be probability distributions on $Z_{p}$ while $H_{\infty}\left(P_{i}\right) \geqq$ 
$\log p-\lambda^{\prime}$ holds, where $0 \leqq \lambda^{\prime} \leqq \log p$. If $x_{i} \stackrel{P_{i}}{\leftarrow} Z_{p}$ (for $i=1$, 2 , $\ldots, n)$ are independent, we have $\operatorname{Pr}\left[F\left(x_{1}, x_{2}, \ldots, x_{n}\right)=0\right] \leqq$ $(d / p) 2^{\lambda^{\prime}}$.

Corollary 1. If $\lambda^{\prime}<\log p-\omega(\log \log p)$, then $\operatorname{Pr}\left[F\left(x_{1}, x_{2} \ldots\right.\right.$, $\left.\left.x_{n}\right)=0\right]$ is negligible (in $\log p$ ).

\section{Framework and Security Notions}

In this section, we define the framework and security notions of leakage-resilient certificateless signature (LR-CLS) schemes under the continual leakage model.

Al-Riyami and Paterson [1] presented the concept of the certificateless public key setting (CL-PKS) and proposed a concrete certificateless signature (CLS) scheme. In CL-PKS, the key generation center (KGC) with a system secret key is responsible to produce the user's initial key, while the user randomly chooses a secret key and computes the corresponding public key. However, formal security notions of CLS schemes were not given until the work of Yum and Lee [46] and Huang et al. [22]. Later, $\mathrm{Hu}$ et al. [19] enhanced the definitions in $[22,46]$ to permit stronger queries for adversaries. Since then, Hu et al.'s security model formalizes the security notions of CLS schemes. In this model, there are two kinds of adversaries, namely, Type I (outsider), Type II (honest-but-curious KGC). A Type I adversary $A_{I}$ acts as an outsider, without the system secret key, who can replace the public key of any entity with another of her/his own choice. In other words, the outsider may obtain the secret key of any entity. A Type II adversary $A_{I I}$ models an honest-but-curious KGC that owns the system secret key, but cannot perform any public key replacement. That is, the honest-but-curious KGC knows the initial key of any entity.

Next, we introduce the so-called stateful from the continual leakage model in [26]. A cryptographic scheme under the continual leakage model is called stateful if the private/secret key must be updated before (or after) executing the cryptographic algorithm while the associated public key remains fixed. To be stateful, each private/secret key must be divided into two parts and stored in different parts of the memory. Hence, for a CLS scheme, we separate the initial key extract algorithm, as well as the signing algorithm, into two steps. In addition, the system secret key and user's private key are separated into two parts, respectively. That is, the two steps of the signing algorithm are carried out by the two parts of the private key, respectively, while the two steps of the initial key extract algorithm are carried out by the two parts of the system secret key.

\subsection{Framework of LR-CLS}

Following Hu et al.'s framework and security notions for CLS schemes, we define a new framework of LR-CLS schemes under the continual leakage model. A LR-CLS scheme consists of the following seven algorithms:

- Setup: This algorithm is run by the key generation center (KGC) that takes a security parameter as input, and outputs the first system secret key $\left(S_{0,1}\right.$, $S_{0,2}$ ) and the public parameters $P P . P P$ is made public and available for all the other algorithms.

_ Initial key extract: The KGC is responsible to run this algorithm which consists two sub-algorithms Extract-1 and Extract-2. For the $i$-th round along with a user's identity ID, the KGC uses the current system secret key $\left(S_{i-1,1}, S_{i-1,2}\right)$ to generate the first initial key $\left(D I D_{0}, Q I D\right)$ of the user while updating the current system secret key with $\left(S_{i, 1}, S_{i, 2}\right)$. Two sub-algorithms are defined as follows:

_ Extract-1: Given $S_{i-1,1}$ and the user's identity $I D$, the algorithm chooses a random number $\gamma$, and outputs $S_{i, 1}$, temporary information $T I_{I E}$ and QID.

_ Extract-2: Given $S_{i-1,2}$ and $T I_{I E}$, the algorithm outputs $S_{i, 2}$ and $D I D_{0}$.

The PKG then sends the initial key $\left(D I D_{0}, Q I D\right)$ to the user.

_ Set secret value: A user with identity ID runs this algorithm to set the secret key of the user. The algorithm randomly selects a secret key $S I D_{0}$, computes the partial public key RID, and then returns $S I D_{0}$ and RID.

_ Set private key: This deterministic algorithm is run by a user with identity $I D$ and takes as input the user's initial key $\left(D I D_{0}, Q I D\right)$ and secret key $S I D_{0}$, and returns the user's private key $\left(\left(D I D_{0,1}, D I D_{0,2}\right)\right.$, $\left.\left(S I D_{0,1}, S I D_{0,2}\right)\right)$.

_ Set public key: This deterministic algorithm is 
run by a user with identity $I D$ and takes as input the user's initial key $\left(D I D_{0}, Q I D\right)$ and the partial public key $R I D$, and returns the user's public key $P I D=(Q I D, R I D)$.

_ Sign: A user with identity ID runs this algorithm which consists of two sub-algorithms Sign-1 and Sign-2. For the $j$-th Sign round, the user employs the current private key $\left(D I D_{j-1}=\left(D I D_{j-1,1}, D I D_{j-1,2}\right)\right.$, $\left.S I D_{j}=\left(S I D_{j-1,1}, S I D_{j-1,2}\right)\right)$ to generate a signature $\sigma$ while updating the current private key with $\left(D I D_{j}=\left(D I D_{j, 1}, \quad D I D_{j, 2}\right), S I D_{j}=\left(S I D_{j, 1}, S I D_{j, 2}\right)\right)$. Two sub-algorithms are presented as follows:

_ Sign-1: Given $D I D_{j-1,1}$ and $S I D_{j-1,1}$ of the current private key and a message $m$, the algorithm chooses a random number $\eta$, and outputs $D I D_{j, 1}$, $S I D_{j, 1}$ and the temporary information $T I_{S}$.

_ Sign-2: Given $D I D_{j-1,2}$ and $S I D_{j-1,2}$ of the user's current private key and the temporary information $T I_{S}$, the algorithm outputs $D I D_{j, 2}, S I D_{j, 2}$ and a signature $\sigma$.

- Verify: This deterministic algorithm takes as input a message $m$, a signature $\sigma$, a user identity $I D$ with $P I D$, and outputs either "accept" or "reject".

\subsection{Security Notions of LR-CLS}

In the presence of the continual leakage model, an adversary $A$ can get leakage information from four sub-algorithms, namely, Extract-1, Extract-2, Sign-1 and Sign-2. In order to represent the leakage information, we use two leakage functions $f_{I E, i}$ and $h_{I E, I}$, respectively, to model the adversary's ability in $E x$ tract-1 and Extract-2 of the $i$-th Initial key extract round. Meanwhile, two leakage functions $f_{S, j}$ and $h_{S, j}$ are used to model the adversary's ability in Sign-1 and Sign-2 of a user's $j$-th Sign round. Note that four leakage functions $f_{I E, i,} h_{I E, i}, f_{S, j}$ and $h_{S, j}$ can be efficiently computed with bounded output length $\{0,1\}^{\lambda}$ ( $\lambda$ is the leakage parameter), namely, $\left|f_{I E, i}\right|,\left|h_{I E, i}\right|,\left|f_{S, j}\right|,\left|h_{S, j}\right| \leq \lambda$, where $\mid$ func $\mid$ denotes the output length of the function func. The outputs of four leakage functions are defined as follows.

- $\Lambda f_{I E, i}=f_{I E, i}\left(S_{i-1,1}\right.$, parameters $)$.

- $\Lambda h_{I E, i}=h_{I E, i}\left(S_{i-1,2}, T I_{I E}\right.$, parameters $)$.

_ $\Lambda f_{S, j}=f_{S, j}\left(D I D_{j-1,1}, S I D_{j-1,1}\right.$, parameters $)$.

- $\Lambda h_{S, j}=h_{S, j}\left(D I D_{j-1,2}, S I D_{j-1,2}, T I_{S}\right.$, parameters $)$.

Here, parameters are the random values involved in the computation of each Extract and Sign round. Note that $T I_{I E}$ and $T I_{S}$ are the outputs of Extract-1 and Sign-1, respectively.

In the LR-CLS scheme under the continual leakage model, the security notions include two kinds of attackers, namely, Type I attacker (outsider) and Type II attacker (honest-but-curious KGC). Both kinds of attackers are extended from the security notions of traditional certificateless signature (CLS) schemes $[19,22,46]$ by adding the key leakage queries. In such a scheme, the system secret key is used to generate the user's initial key by the KGC and the user's private key is used to generate the signature by the signer. Hence, under the continual leakage model, LR-CLS schemes are allowed to leak partial information of the system secret key in the Initial key extract phase and the user's private key in the Sign phase.

The adversary model of LR-CLS schemes under the continual leakage model consists of two kinds of adversaries, namely, Type I (outsider), Type II (honest-but-curious KGC).

- Type I adversary (outsider): An adversary of this type cannot access the system secret key, but she/ he can replace the public key of any entity with another of her/his own choice. In other words, the adversary may obtain the secret key of any entity. Meanwhile, the adversary may obtain not only the leakage information of a user's initial key of the private key in the Sign phase, but also the leakage information of the KGC's system secret key in the Initial key extract phase.

- Type II adversary (honest-but-curious KGC): An adversary of this type is an honest-but-curious KGC who has access to the system secret key, but cannot perform any public key replacement. That is, the honest-but-curious KGC knows the initial key of any entity while obtaining the leakage information of a user's secret key of the private key in the Sign phase.

In the following, we employ a security game to model security notions of LR-CLS schemes under the continual leakage model. The security game describes the interactions between a challenger and an adversary.

Definition 1. A LR-CLS scheme possesses existential unforgeability against adaptive chosen-message attacks under continual leakage model (UF-LR-CLSACMA) if no probabilistic polynomial-time adver- 
sary $A$ (including Types I and II adversaries) has a non-negligible advantage in the following UF-LRCLS-ACMA game played with a challenger $C$. The advantage of the adversary $A$ is defined as the probability that $A$ wins the games. Such an adversary $A$ is referred as an UF-LR-CLS-ACMA adversary.

- Setup. The challenger $C$ takes as input a security parameter and runs the Setup algorithm to produce the first system secret key $\left(S_{0,1}, S_{0,2}\right)$ and a list of public parameters $P P$. $P P$ is given to the adversary $A$. Meanwhile, if $A$ is of Type II adversary, $C$ gives the system secret key $\left(S_{0,1}, S_{0,2}\right)$ to the adversary $A$. If $A$ is of Type I adversary, the system secret key $\left(S_{0,1}, S_{0,2}\right)$ is kept secret by the challenger $C$.

- Queries. The adversary $A$ can adaptively make numerous queries to the challenger $C$ as follows.

- Initial key extract query(ID). For the $i$-th Extract round, upon receiving this query along with a user's identity $I D$, the challenger $C$ uses the current system secret key $\left(S_{i-1,1}, S_{i-1,2}\right)$ to generate the first initial key $\left(D I D_{0}, Q I D\right)$ of the user while updating the current system secret key with $\left(S_{i, 1}\right.$, $S_{i, 2}$ ) by running two sub-algorithms Extract-1 and Extract-2. Finally, $C$ sends $\left(D I D_{0}, Q I D\right)$ to $A$.

_ Initial key extract leak query $\left(f_{I E, i,}, h_{I E, i}, i\right)$ : For the $i$-th Extract query, $A$ can issue the Initial key extract leak query only once by providing two leakage functions $f_{I E, i}$ and $h_{I E, i}$. $C$ computes the leakage information of $\left(\Lambda f_{I E, i}, \Lambda h_{I E, i}\right)$ and sends it to $A$. Here we assume that two leakage functions $f_{I E, i}$ and $h_{I E, i}$ can be efficiently computed with bounded length output in $\{0,1\}^{\lambda}$, namely, $\left|f_{I E, i}\right|,\left|h_{I E, i}\right| \leq \lambda$.

_ Public key retrieve query (ID). When $A$ issues this query along with an identity $I D$, the challenger $C$ returns the corresponding public key $P I D=(Q I D, R I D)$ to $A$.

_ Public key replace query $\left(I D, P I D^{\prime}=\left(Q I D^{\prime}, R I D^{\prime}\right)\right)$. Upon receiving this query, the user's original public key is replaced with $P I D^{\prime}=\left(Q I D^{\prime}, R I D^{\prime}\right)$ and the challenger $C$ records the replacement.

_ Secret key extract query (ID). When $A$ issues this query along with an identity $I D$, the challenger $C$ returns the secret key $S I D_{0}$. Here, the query is forbidden if the identity ID has already appeared in the public key replace query.

_ Sign query (ID, $m$ ). For the $j$-th Sign round, upon receiving this query along with a user's iden- tity $I D$ and a message $m$, the challenger $C$ uses the user's current private key $\left(D I D_{j-1}=\left(D I D_{j-1,1}\right.\right.$, $\left.\left.D I D_{j-1,2}\right), S I D_{j}=\left(S I D_{j-1,1}, S I D_{j-1,2}\right)\right)$ to produce a signature $\sigma$ on the message $m$ by running two sub-algorithms Sign-1 and Sign-2 while updating the current private key with $\left(D I D_{j}=\left(D I D_{j, 1}\right.\right.$, $\left.\left.D I D_{j, 2}\right), S I D_{j}=\left(S I D_{j, 1}, S I D_{j, 2}\right)\right)$. The challenger $C$ then returns $\sigma$ to $A$.

_ Sign leak query $\left(f_{S, j}, h_{S, j}, j\right)$ : For the $j$-th Sign query of the user with identity $I D$, the adversary $A$ can issue the Sign leak query only once by providing two leakage functions $f_{S, j}$ and $h_{S, j}$. After receiving this query, the challenger $C$ computes and sends the leakage information $\left(\Lambda f_{S, j}, \Lambda h_{S, j}\right)$ to $A$, where $f_{S, j}$ and $h_{S, j}$ can be efficiently computed with bounded length output in $\{0,1\}^{\lambda}$. Meanwhile, an adversary of Type II (honest-but-curious KGC) knows the initial key of any entity so that $\left(\Lambda f_{S, j}\right.$, $\Lambda h_{S, j}$ ) includes only the leakage information of a user's secret key $\left(S I D_{j-1,1}, S I D_{j-1,2}\right)$ of the private key. An adversary of Type I (outsider) can obtain the leakage information of a user's initial key $\left(D I D_{j-1,1}, D I D_{j-1,2}\right)$ of the private key since an outsider owns the secret key of any entity.

- Forgery. The adversary $A$ generates a tuple ( $m^{*}, I D^{*}$, $\left.\sigma^{*}, P I D^{*}=\left(Q I D^{*}, R I D^{*}\right)\right)$. We say that $A$ wins the game if the following conditions are satisfied.

1 The response of the Verify algorithm on ( $m^{*}, I D^{*}$, $\sigma^{*}, P I D$ ) is "accept".

$2\left(m^{*}, I D^{*}\right)$ has never been issued during the Sign query.

3 If $A$ is of Type I adversary (outsider), $I D^{*}$ has never been issued during the Initial key extract query. If $A$ is of Type II adversary (honest-but-curious KGC), it is disallowed to issue the queries on the public key replace query and secret key extract query on ID*.

\section{The Proposed LR-CLS Scheme}

Based on the leakage-resilient signature scheme in [18] and the leakage-resilient ID-based signature scheme in [42], we present the first LR-CLS scheme, as defined in Section 3.1, which consists of seven algorithms. Fig. 1 depicts the key generation processes of the KGC and users. The functionalities of the Sign 
and Verify algorithms are depicted in Figure 2. The details of seven algorithms are given as follows.

- Setup: The KGC chooses two multiplicative cyclic groups $G$ and $G_{T}$ of sufficiently large prime order $p$ while picking an arbitrary generator $g$ of the group $G$. Let $e: G \times G \rightarrow G_{\mathrm{T}}$ be an admissible bilinear pairing. The KGC runs the following steps:

1 Pick a random value $x \in Z_{p}^{*}$, and compute $X=g^{x}$ and $X_{T}=e\left(g^{x}, g\right)$.

2 Pick a random value $\alpha \in Z_{p}^{*}$ and set the first system secret key $\left(S_{0,1}, S_{0,2}\right)=\left(g^{\alpha}, X \cdot g^{-\alpha}\right)$.

3 Pick four values $u i_{0}, u i_{1}, m i_{0}, m i_{1} \in Z_{p}^{*}$ at random, and compute $U_{0}=g^{u i_{0}}, U_{1}=g^{u i_{1}}, M_{0}=g^{m i_{0}}$ and $M_{1}=g^{m i_{1}}$.

4 Publish the public parameters $P P=\left(G, G_{T}, e, p, g\right.$, $\left.X_{T}, U_{0}, U_{1}, M_{0}, M_{1}\right)$.

- Initial key extract: For the $i$-th round along with a user's identity $I D$, the KGC uses the current system secret key $\left(S_{i-1,1}, S_{i-1,2}\right)$ to generate the first initial key $\left(D I D_{0}, Q I D\right)$ of the user while updating the current system secret key with $\left(S_{i, 1}, S_{i, 2}\right)$ by running two sub-algorithms Extract-1 and Extract-2 as follows:

_ Extract-1: Given the user's identity ID, the KGC uses $S_{i-1,1}$ to generate the temporary information and QID as follows.

1 Randomly select two values $\gamma, a \in Z_{p}^{*}$.

2 Compute $Q I D=\mathrm{g}^{\prime \prime}$ and $S_{i, 1}=S_{i-1,1} \cdot g^{a}$.

3 Compute the temporary information $T I_{I E}=$ $S_{i, 1} \cdot\left(U_{0} \cdot U_{1}^{I D}\right)^{y}$.

- Extract-2: Given $T I_{I E}$, the KGC uses $S_{i-1,2}$ to generate $D I D_{0}$ as follows.

1 Compute $S_{i, 2}=S_{i-1,2} \cdot g^{-a}$.

2 Set $D I D_{0}=S_{i, 2} \cdot T I_{I E}$.

Finally, the KGC updates the current system secret key by $\left(S_{i, 1}, S_{i, 2}\right)$ and sends the first initial key $\left(D I D_{0}, Q I D\right)=\left(X \cdot\left(U_{0} \cdot U_{1}^{I D}\right)^{\gamma}, \mathrm{g}^{\prime \prime}\right)$ to the user via a secure channel. Meanwhile, the user can validate the correctness of the first initial key by checking $e\left(g, D I D_{0}\right)=X_{T} \cdot e\left(Q I D, U_{0} \cdot U_{1}^{I D}\right)$.

_ Set secret value: A user with identity ID randomly selects a number $z \in Z_{p}^{*}$, and computes the secret key $S I D_{0}=g^{z}$ and the partial public key $R I D=e\left(g^{z}, g\right)$.

- Set private key: Given the initial key $\left(D I D_{0}\right.$, $\mathrm{QID})=\left(X \cdot\left(U_{0} \cdot U_{1}^{I D}\right)^{\gamma}, \mathrm{g}^{\prime}\right)$ and the secret key $S I D_{0}=g^{z}$, the user with identity $I D$ chooses two random numbers $\beta, \omega \in Z_{p}^{*}$ and sets her/his current private key $\left(\left(D I D_{0,1}, D I D_{0,2}\right)=\left(g^{\beta}, D I D_{0} \cdot g^{-\beta}\right),\left(S I D_{0,1}, S I D_{0,2}\right)=\right.$ $\left.\left(g^{w}, S I D_{0} \cdot g^{-w}\right)\right)$.

- Set public key: Given the initial key $\left(D I D_{0}\right.$, $Q I D)=\left(X \cdot\left(U_{0} \cdot U_{1}^{I D}\right)^{\gamma}, \mathrm{g}^{\prime \prime}\right)$ and the partial public key $R I D=e\left(g^{z}, g\right)$, the user with identity $I D$ sets her/his public key $P I D=\left(Q I D=\mathrm{g}^{\prime \prime}, R I D=e\left(g^{z}, g\right)\right)$.

- Sign: For the $j$-th round of the signer with identity $I D$, given a message $m$, the signer employs the current private key $\left(\left(D I D_{j-1,1}, D I D_{j-1,2}\right),\left(S I D_{j-1,1}\right.\right.$, $\left.S I D_{j-1,2}\right)$ ) to generate a signature $\sigma$ while updating the current private key to $\left(D I D_{j}=\left(D I D_{j, 1}, D I D_{j, 2}\right)\right.$, $\left.S I D_{j}=\left(S I D_{j, 1}, S I D_{j, 2}\right)\right)$. The signer runs two subalgorithms as follows:

_ Sign-1: Given the message $m$, the signer uses $D I D_{j-1,1}$ and $S I D_{j-1,1}$ to generate the temporary information $T I_{S}$ and compute new $D I D_{j, 1}$ and $S I D_{j, 1}$ by the following steps:

1 Choose three random numbers $b, c, \eta \in Z_{p}^{*}$.

2 Compute $D I D_{j, 1}=D I D_{j-1,1} \cdot g^{b}$ and $S I D_{j, 1}=S I D_{j-1,1} \cdot g^{c}$.

3 Compute the temporary information $T I_{S}=$ $S I D_{j, 1} \cdot D I D_{j, 1} \cdot\left(M_{0} \cdot M_{1}^{m}\right)^{\eta}$.

4 Compute $\sigma_{2}=g^{n}$.

- Sign-2: Given $T I_{S}$, the signer uses $D I D_{j-1,2}$ and $S I D_{j-1,2}$ to generate a signature $\sigma$ and compute new $D I D_{j, 2}, S I D_{j, 2}$ by the following steps:

1 Compute $D I D_{j, 2}=D I D_{j-1,2} \cdot g^{-b}$ and $S I D_{j, 2}=S I D_{j-1,2} \cdot g^{c}$.

2 Compute $\sigma_{1}=S I D_{j, 2} \cdot D I D_{j, 2} \cdot T I_{S^{*}}$

Finally, the signer outputs a signature $\sigma=\left(\sigma_{1}, \sigma_{2}\right)$.

Verify: Given a signature $\sigma=\left(\sigma_{1}, \sigma_{2}\right)$ on the message $m$ for the signer with identity $I D$ and public key $P I D=\left(Q I D=\mathrm{g}^{\prime \prime}, R I D=e\left(g^{z}, g\right)\right)$, a verifier accepts the signature if $e\left(g, \sigma_{1}\right)=R I D \cdot X_{T} \cdot e\left(Q I D, U_{0} \cdot U_{1}^{I D}\right)$. $e\left(\sigma_{2}, M_{0} \cdot M_{1}{ }^{m}\right)$; or rejects it otherwise. In the following, we show the correctness of the verifying equality as follows.

$$
\begin{aligned}
& e\left(g, \sigma_{1}\right) \\
& =e\left(g, S_{j, 2} \cdot D I D_{j, 2} \cdot S I D_{j, 1} \cdot D I D_{j, 1} \cdot\left(M_{0} \cdot M_{1}^{m}\right)^{\eta}\right) \\
& =e\left(g, S_{j, 2} \cdot S I D_{j, 1} \cdot D I D_{j, 2} \cdot D I D_{j, 1} \cdot\left(M_{0} \cdot M_{1}^{m}\right)^{\eta}\right) \\
& =e\left(g, g^{z} \cdot X \cdot\left(U_{0} \cdot U_{1}^{I D}\right)^{\gamma} \cdot\left(M_{0} \cdot M_{1}^{m}\right)^{\eta}\right) \\
& =e\left(g, g^{z} \cdot g^{x} \cdot\left(U_{0} \cdot U_{1}^{I D}\right)^{\gamma} \cdot\left(M_{0} \cdot M_{1}^{m}\right)^{\eta}\right) \\
& =e\left(g, g^{z}\right) \cdot e\left(g, g^{x}\right) \cdot e\left(g,\left(U_{0} \cdot U_{1}^{I D}\right)^{\eta}\right) \cdot e\left(g,\left(M_{0} \cdot M_{1}^{m}\right)^{\eta}\right) \\
& =e\left(g^{z}, g\right) \cdot e\left(g^{x}, g\right) \cdot e\left(g^{\eta},\left(U_{0} \cdot U_{1}^{I D}\right)\right) \cdot e\left(g^{\eta},\left(M_{0} \cdot M_{1}^{m}\right)\right) \\
& =R I D \cdot X_{T} \cdot e\left(Q I D, U_{0} \cdot U_{1}^{I D}\right) \cdot e\left(\sigma_{2}, M_{0} \cdot M_{1}^{m}\right) .
\end{aligned}
$$




\section{Figure 1}

The key generation processes of the KGC and users

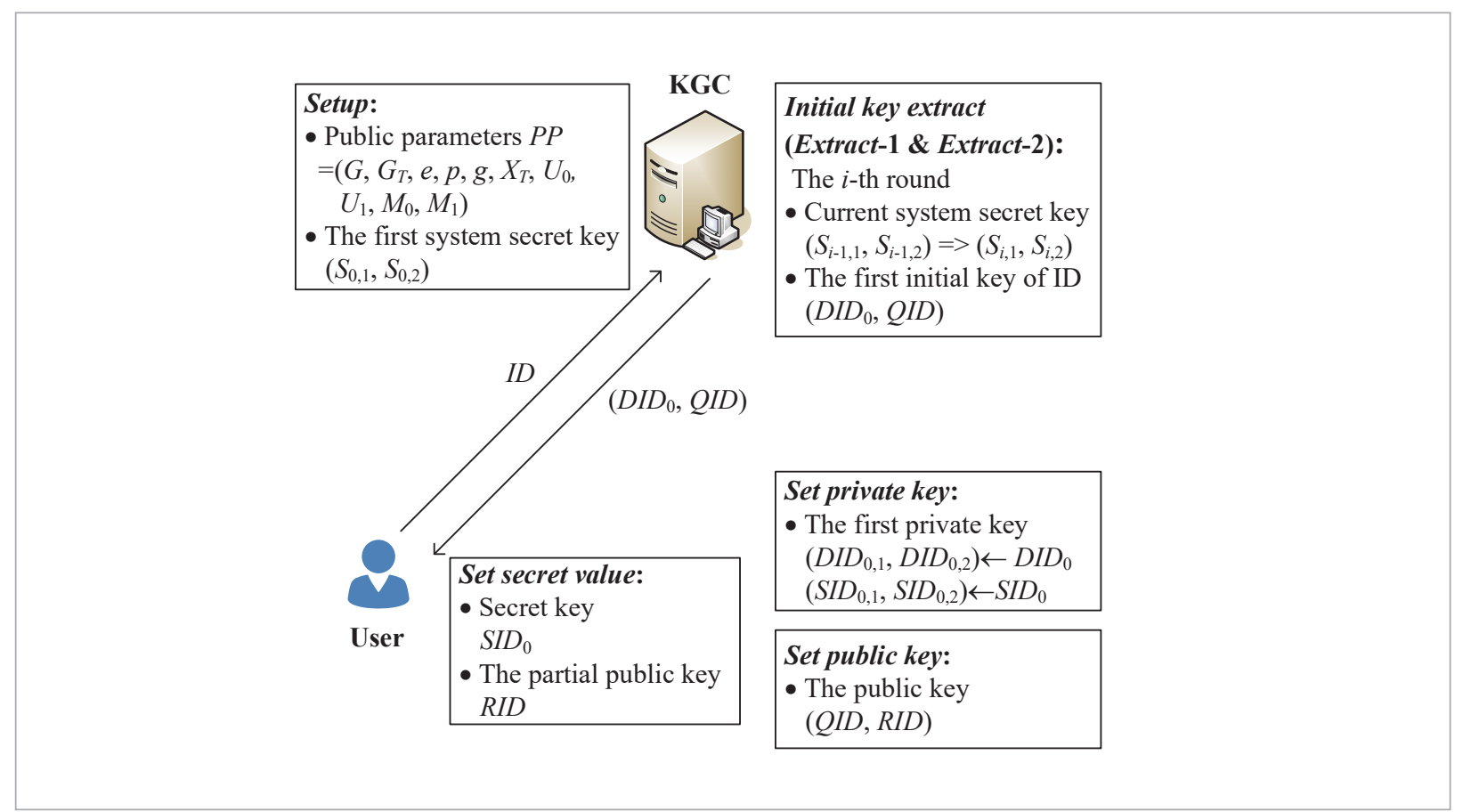

Figure 2

The Sign and Verify algorithms of the proposed scheme

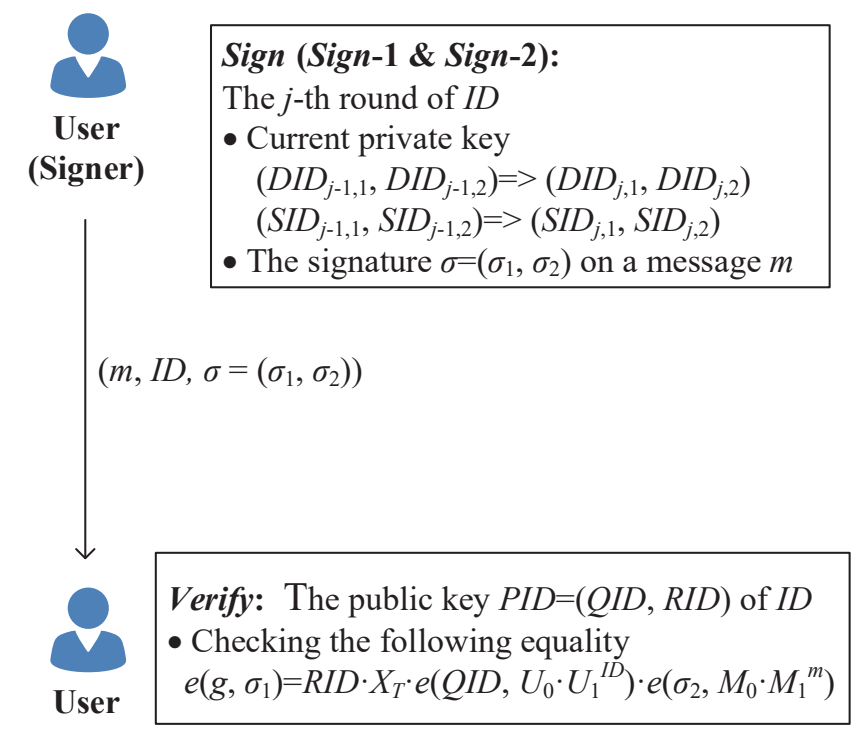

(Verifier) 


\section{Security Analysis}

In the proposed LR-CLS scheme, a user's private key consists of two components, namely, an initial key and a secret key. As the aforementioned UF-LRCLS-ACMA game in Definition 1, there are two kinds of adversaries, which include Type I (outsider) and Type II (honest-but-curious KGC). In the generic bilinear group model, we demonstrate that our LR-CLS scheme possesses existential unforgeability against adaptive chosen-message attacks for both Type I and Type II adversaries under the continual leakage model. We first prove that the non-leakage version of our LR-CLS scheme without leakage queries, denoted by $\Pi_{N L}$, is UF-CLS-ACMA secure in the generic bilinear group model. Then, based on the security of the non-leakage version, we demonstrate that our proposed LR-CLS scheme under the continual leakage model is UF-LR-CLS-ACMA secure in the generic bilinear group model.

The non-leakage version $\Pi_{N L}$ of our LR-CLS scheme consists of seven algorithms Setup ${ }_{N L}$, Initial key extract $_{N L}$, Set secret value ${ }_{N L}$, Set private key ${ }_{N L}$,Set public key $_{N L}$, Sign $_{N L}$ and Verify $y_{N L}$ :

_ Setup $_{N L}$ : In this algorithm, the system key is generated by $X=g^{x}$ where $x$ is picked from $Z_{p}^{*}$ randomly. The generation of the public parameters $P P=\left(G, G_{T}, e, p, g, X_{T}, U_{0}, U_{1}, M_{0}, M_{1}\right)$ is identical to that of the proposed LR-CLS scheme. At the end of this algorithm, the KGC publishes the public parameters $P P$.

_ Initial key extract ${ }_{N L}$ : Upon receiving a user's identity $I D$, the KGC uses the system key $X$ to generate the user's initial key (DID).

_,$Q I D)=\left(X \cdot\left(U_{0} \cdot U_{1}^{I D}\right)^{\gamma}, \mathrm{g}^{\prime \prime}\right)$ where $\gamma$ is a random number picked from $Z_{p}^{*}$. The KGC then sends the user's private key pair ( $D I D, Q I D)$ to the user via a secure channel.

_ Set secret value $_{N L}:$ A user with identity ID randomly selects a random number $z \in Z_{p}^{*}$ and computes the secret key $S I D=g^{z}$ and the partial public key $R I D=e\left(g^{z}, g\right)$.

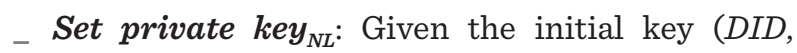
$Q I D)=\left(X \cdot\left(U_{0} \cdot U_{1}^{I D}\right)^{\gamma}, \mathrm{g}^{\prime}\right)$ and the secret key $S I D=g^{z}$, the user with identity $I D$ sets her/his private key $(D I D, S I D)=\left(X \cdot\left(U_{0} \cdot U_{1}^{I D}\right)^{\gamma}, g^{z}\right)$.

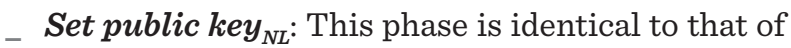
the proposed LR-CLS scheme.
- $\boldsymbol{S i g n _ { N L }}$ : For the signer with identity ID, given a message $m$, the signer employs the user's private key DID and the user's secret key SID to generate a signature $\sigma=\left(\sigma_{1}, \sigma_{2}\right)=\left(\operatorname{SID} \cdot D I D \cdot\left(M_{0} \cdot M_{1}^{m}\right)^{\eta}, g^{\eta}\right)$, where $\eta \in Z_{p}^{*}$. The signer then outputs $\sigma$.

- Verify $y_{N L}$ : Upon receiving the signature $\left(\sigma_{1}, \sigma_{2}\right)$, a verifier accepts the signature if $e\left(g, \sigma_{1}\right)=R I D \cdot X_{T} \cdot e\left(Q I D, U_{0} \cdot U_{1}^{I D}\right) \cdot e\left(\sigma_{2}, M_{0} \cdot M_{1}{ }^{m}\right)$, where QID and RID are the public keys of the user with identity $I D$; or rejects it otherwise.

In the generic bilinear group (GBG) model, we first prove that our non-leakage version $\Pi_{N L}$ is UF-CLSACMA secure against Type I and Type II adversaries in Theorems 1 and 2, respectively. Based on the security of the non-leakage version, by adding extra leak queries, we then prove that our LR-CLS scheme under the continual leakage model is UF-LR-CLSACMA secure against Type I and Type II adversaries in Theorems 3 and 4, respectively. Figure. 3 demonstrates the relationships of the associated four security theorems. In addition, Figure 4 depicts the conceptual principle of the security games $g_{N L-I}$ and $g_{N L-I I}$ employed in Theorems 1 and 2.

Theorem 1. In the generic bilinear group model, the non-leakage version $\Pi_{N L}$ of the proposed LR-CLS scheme is provably secure against the Type I adversary (outsider).

Proof: Let $A_{N L-I}$ be a Type I adversary who can break the non-leakage CLS scheme $\Pi_{N L}$ while $A_{N L-\text { I }}$ is allowed to issue all the queries at most $q$ times. The advantage of $A_{N L-\mathrm{I}}$ is defined as the probability that $A_{N L \text {-I }}$ wins the following game $g_{N L \text {-I }}$ played with a challenger $C$.

Game $\boldsymbol{g}_{N L-\mathrm{I}}$ In the game $g_{N L-\mathrm{I}}$, there are three phases, Setup, Queries and Forgery phases. At the end of this game, $A_{N L \text {-I }}$ outputs a forgery signature. In Queries phase, $A_{N L-\mathrm{I}}$ may issue eight kinds of queries in any order at most $q$ times. Three phases are described as below:

Setup phase: The challenger $C$ builds and maintains two lists $L_{G}$ and $L_{T}$ which are used to record group elements in $G$ and $G_{T}$, respectively, described below.

_ The list $L_{G}$ consists of pairs of the form $\left(F_{G, \mathrm{w}, k, l}, \quad \xi_{G, \mathrm{w}, k, l}\right)$, where $F_{G, \mathrm{w}, k, l}$ is a multivariate polynomial with coefficients in $Z_{p}$ and variates in $G$ and $\xi_{G, \mathrm{w}, k, l}$ is the bit string denoting $F_{G, \mathrm{w}, k, l,}$. The first index of $F_{G, \mathrm{w}, k, l}$ is " $G$ ", which denotes this elements represents an element in $G$, on 


\section{Figure 3}

The relationships of four security theorems

\section{Type I adversary (outsider)}

\section{Theorem 1:}

The security of the non-leakage version $\Pi_{N L}$ of the proposed LR-CLS Scheme

- In the generic bilinear group model

Extra leakage queries:

- Initial key extract leak query

- Sign leak query

Theorem 3:

The security of the proposed LR-CLS scheme $\Pi_{L R}$

- In the generic bilinear group model

- Under the continual leakage model

\section{Type II adversary (honest- but-curious KGC)}

Theorem 2:

The security of the non-leakage version $\Pi_{N L}$ of the proposed LR-CLS scheme

- In the generic bilinear group model

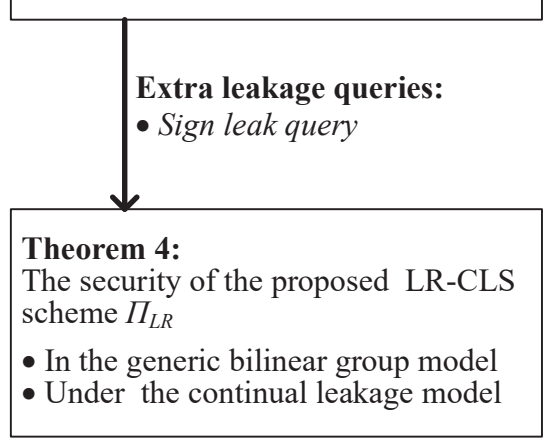

Figure 4

The conceptual principle of the security games $g_{N L-I}$ and $g_{N L-I I}$ in Theorems 1 and 2

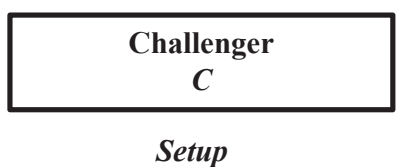

$P P$ and Lists:

- $L_{G}$ : elements in $G$

- $L_{T}$ : elements in $G_{T}$

- $L_{I K}$ : tuples of initial keys

- $L_{S K}$ : tuples of secret keys

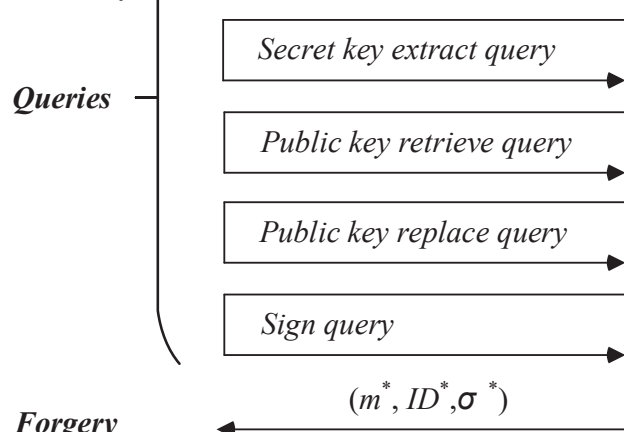

The probabilities of two cases:

- There exists a collision in group $G$ or $G_{T}$

- $\left(m^{*}, I D^{*}, \sigma^{*}\right)$ is a valid forged signature

$P P$

Group and Pairing oracles

\section{Initial key extract query}

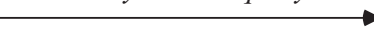

Public key retrieve query

$\left(m, 1 D^{*}, \sigma *\right)$

\section{Adversary \\ $A_{N L-\mathrm{I}}$ or $A_{N L \text {-II }}$}

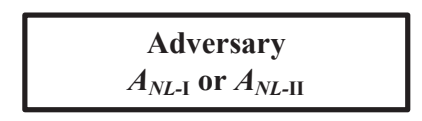

Type I (outsider, $\left.A_{N L-\mathrm{I}}\right)$ :

Allow to issue all queries except for the Initial key extract query on the target $I D^{*}$

Type II (KGC, $\left.A_{N L-I I}\right)$ :

Allow to issue all queries except for the Secret key extract and Public key replace query on the target $I D^{*}$

$A_{N L \text {-I }}$ or $\boldsymbol{A}_{N L \text {-II }}$

- A forged signature $\left(m^{*}, I D^{*}, \sigma^{*}\right)$ 
the other hand if the first index is " $T$ ", which denotes this elements in $G_{T}$. The second index "w" is indicating the type of query. The third and fourth index " $k$ " and "l" represent the $l$-th element appeared in the $k$-th type "w" query in this game. Meanwhile, six initial tuples ( $g$, $\left.\xi_{G, I, 1,1}\right),\left(X, \xi_{G, I, 1,2}\right),\left(U_{0}, \xi_{G, I, 1,3}\right),\left(U_{1}, \xi_{G, I, 1,4}\right),\left(M_{0}, \xi_{G, I, 1,5}\right)$ and $\left(M_{1}, \xi_{G, I, 1,6}\right)$ are added in $L_{G}$, where $\xi_{G, I, 1, i}$ (for $i=1,2, \ldots, 6)$ are six different bit strings generated randomly representing the elements in $G$.

The List $L_{T}$ consists of pairs of the form $\left(F_{T, \mathrm{w}, k, l}\right.$, $\left.\xi_{T, \mathrm{w}, k, l}\right)$. The meanings of the indexes of $F_{T, \mathrm{w}, k, l}$ are the same with the descriptions of $F_{G, \mathrm{w}, k, l}$ before. The only difference is that $F_{T, w, k, l}$ is a multivariate polynomial with coefficients in $Z_{p}$ and variates in $G$ or $G_{T}$. The initial tuple $\left(X_{T}, \xi_{T, I, 1,1}\right)$ is added in $L_{T}$, where $\xi_{T, \mathrm{w}, k, l}$ is a bit string generated randomly representing $X_{T}$. The challenger sends the bit strings of the public parameters to $A_{N L-\mathrm{I}}$ at the end of this phase.

Moreover, the challenger $C$ also maintains two lists $L_{I K}$ and $L_{S K}$ to record the tuples of the users' initial keys and secret keys, respectively. More precisely, $L_{I K}$ and $L_{S K}$ consists, respectively, of tuples of the forms (ID, DID, QID) and (ID, SID, RID), where ID is in $Z_{p}^{*}$. Here, DID, QID, SID and RID are multivariate polynomials.

Queries phase: In this phase, the adversary $A_{N L-\mathrm{I}}$ may issue eight kinds of queries to the challenger $C$ at most $q$ times in any order.

_ Group oracle $O_{G}\left(\xi_{G, 0, i, 1}, \xi_{G, O, i, 2}\right.$, operation): For the $i$-th group oracle $O_{G}$, upon receiving this query along with two bit strings $\xi_{G, O, i, 1}, \xi_{G, O, i, 2}$ and an operation (multiplication or division), $C$ runs the following three steps:

- Translates the bit strings $\xi_{G, O, i, 1}$ and $\xi_{G, O, i, 2}$ back into two polynomials $F_{G, O, i, 1}$ and $F_{G, O, i, 2}$, respectively, in the following way: $C$ tries to find a pair $\left(F_{G, \omega, k, l,}, \xi_{G, \omega, k, l}\right)$ in $L_{G}$ such that $\xi_{G, \omega, k, l}=$ $\xi_{G, O, i, 1}$. If so, $C$ sets $F_{G, O, i, 1}=F_{G, \omega, k, l}$ Otherwise, $C$ randomly chooses a new variate $S_{G, O, i, 1}$ in $G$, sets $F_{G, O, i, 1}=S_{G, O, i, 1}$, and records $\left(F_{G, O, i, 1}, \xi_{G, O, i, 1}\right)$ in $L_{G}$. Similarly, $C$ translates the bit string $\xi_{G, O, i, 2}$ into $F_{G, 0, i, 2}$.

_ Set the polynomial $F_{G, O, i, 3}=F_{G, O, i, 1}+F_{G, O, i, 2}$ if the operation is a multiplication, and $F_{G, O, i, 3}=F_{G, O, i, 1}-$ $F_{G, O, i, 2}$ if the operation is a division.
- Try to find a pair $\left(F_{G, \omega, k, l}, \xi_{G, \omega, k, l}\right)$ in $L_{G}$ such that $F_{G, \omega, k, l}=F_{G, O, i, 3}$. If so, $C$ returns $\xi_{G, \omega, k, l}$ to $A_{N L-\mathrm{I}}$. Otherwise, $C$ randomly selects a bit string $\xi_{G, O, i, 3}$ which is distinct from all the $\xi_{G, \omega, k, l}$ appeared in $L_{G}$. Finally, $C$ records $\left(F_{G, 0, i, 3}\right.$, $\left.\xi_{G, O, i, 3}\right)$ in $L_{G}$ and returns $\xi_{G, \omega, k, l}=\xi_{G, 0, i, 3}$ to $A_{N L-\mathrm{I}}$.

Note that the polynomials $F_{G, O, i, 1}, F_{G, O, i, 2}$ and $F_{G, O, i, 3}$ mentioned above are recorded in the list $L_{G}$.

- Group oracle $O_{T}\left(\xi_{T, O, i, 1}, \xi_{T, O, i, 2}\right.$, operation): This oracle is similar to the Group oracle $O_{G}$ above. For the $i$-th group oracle $O_{T}$, upon receiving this query along with two bit strings $\xi_{T, O, i, 1}, \xi_{T, O, i, 2}$ and an operation (multiplication or division), $C$ returns $\xi_{T, \omega, k, l}=\xi_{T, O, i, 3}$ to $A_{N L \text {-I }}$ and the polynomials $F_{T, O, i, 1}, F_{T, O, i, 2}$ and $F_{T, O, i, 3}$ are recorded in $L_{T}$ after this query.

Pairing oracle $O_{P}\left(\xi_{G, P, i, 1}, \xi_{G, P, i, 2}\right):$ For the $i$-th pairing oracle $O_{P}$, upon receiving this query along with two bit strings $\xi_{G, P, i, 1}, \xi_{G, P, i, 2}, C$ runs the following steps:

- Similarly as in the Step 1 of the Group oracle $O_{G}, C$ translates the bit strings $\xi_{G, P, i, 1}$ and $\xi_{G, P, i, 2}$ back into two polynomials $F_{G, P, i, 1}$ and $F_{G, P, i, 2,}$, respectively. Additionally, $C$ computes the polynomial $F_{T, P, i, 1}=F_{G, P, i, 1} \cdot F_{G, P, i, 2}$.

- $C$ tries to find a pair $\left(F_{T, \omega, k, l}, \xi_{T, \omega, k, l}\right)$ in $L_{T}$ such that $F_{T, \omega, k, l}=F_{T, P, i, 1}$. If so, $C$ returns $\xi_{T, \omega, k, l}$ to $A_{N L-\mathrm{I}}$. Otherwise, $C$ randomly selects a bit string $\xi_{T, P, i, 1}$ which is distinct from all the $\xi_{T, \omega, k, l}$ appeared in $L_{T}$. Finally, $C$ records $\left(F_{T, P, 1}, \xi_{T, P, 1}\right)$ in $L_{T}$ and returns $\xi_{T, \omega, k, l}=\xi_{T, P, i, 1}$ to $A_{N L-\mathrm{I}}$.

It is worth mentioning, after this query, that the polynomials $F_{G, O, i, 1}$ and $F_{G, O, i, 2}$ have been recorded in the list $L_{G}$ while $F_{T, O, i, 1}$ has also been recorded in the list $L_{T}$.

- Initial key extract query $\mathbf{Q}_{I E}\left(I D_{I E, i}\right)$ : For the $i$-th initial key extract query, upon receiving this query along with a user's identity $I D_{I E, i} \in Z_{p}^{*}, C$ first checks whether $I D_{I E, i}$ has been recorded in the list $L_{I K}$. If so, $C$ returns the bit strings $\left(\xi_{G, I E, i, 1}\right.$, $\left.\xi_{G, I E, i, 2}\right)$ representing the initial key $(D I D, Q I D)$ of the user with identity $I D_{I E, i}$ to $A_{N L-\mathrm{I}}$. Otherwise, $C$ runs the following steps:

$C$ defines one variate $T_{G, I E, i, 2}$ in $G$ for representing $Q I D$ of the identity $I D_{I E, i}$ and sets $F_{G, I E, i, 2}=T_{G, I E, i, 2}$. Additionally, $C$ selects 
a random bit string $\xi_{G, I E, i, 2}$ which is distinct from all the $\xi_{G, \omega, l,}$ appeared in $L_{G}$, and records $\left(F_{G, I E, i, 2}, \xi_{G, I E, i, 2}\right)$ in $L_{G \cdot}$. Furthermore, $C$ computes the polynomial $F_{G, I E, i, 1}=X+\left(U_{0}+I D_{I E, i} \cdot U_{1}\right) \cdot T_{G, I E ;, i, 2}$ for representing $D I D$ of the identity $I D_{I E, i}$.

- $C$ selects a random bit string $\xi_{G, I E, i, 1}$ which is distinct from all the $\xi_{G, \omega, k, l}$ appeared in $L_{G}$. Finally, $C$ records $\left(F_{G, I E, i, 1}, \xi_{G, I E, i, 1}\right)$ in $L_{G}$ and returns $\left(\xi_{G, I E, i, 1}, \xi_{G, E E, i, 2}\right)$ to $A_{N L-\mathrm{I}}$.

Finally, the challenger $C$ also maintains an element $\left(I D_{I E, i}, F_{G, I E, i, 1}, F_{G, I E, i, 2}\right)$ in the list $L_{I K}$.

- Secret key extract query $Q_{S E}\left(I D_{S E, i}\right)$ : When $A_{N L-I}$ issues the $i$-th Secret key extract query along with an identity $I D_{S E, i}$, the challenger $C$ returns the bit strings $\left(\xi_{T, S E, i, 1}, \xi_{T, S E, i, 2}\right)$ for representing the secret key pair (SID, RID) by running the steps as below.

_ The challenger $C$ checks whether the secret key pair of identity $I D_{S E, i}$ has been recorded in $L_{S K}$. If so, $C$ returns the bit strings $\left(\xi_{G, S E, i, 1}\right.$, $\left.\xi_{T, S E, i, 2}\right)$ representing the secret key $(S I D, R I D)$ of the user with identity $I D_{S E, i}$ to $A_{N L-\mathrm{I}}$.

_ If the identity $I D_{S E, i}$ is not recorded in $L_{S K}, C$ defines one variate $T_{G, S E, i, 1}$ in $G$ and sets the polynomial $F_{G, S E, i, 1}=T_{G, S E, i, 1}$ for representing $S I D$ of $I D_{S E, i}$. Moreover, $C$ randomly chooses a bit string $\xi_{G, S E, i, 1}$ which is distinct from all the $\xi_{G, \omega, k, l}$ appeared in $L_{G}$. Then $C$ records $\left(F_{G, S E, i, 1}\right.$, $\left.\xi_{G, S E, i, 1}\right)$ in $L_{G}$.

_ $C$ sets the polynomial $F_{T, S E, i, 2}=T_{G, S E, i, 1} \cdot g$ for representing $R I D$ for $I D_{S E, i}$. Moreover, $C$ selects a random bit string $\xi_{T, S E, i, 2}$ which is distinct from all the $\xi_{T, \omega, k, l}$ appeared in $L_{T}$. Then $C$ records $\left(F_{T, S E, i, 2}, \xi_{T, S E, i, 2}\right)$ in $L_{T}$ and returns $\left(\xi_{G, S E, i, 1}, \xi_{T, S E, i, 2}\right)$ to $A_{N L-\mathrm{I}}$.

Finally, the challenger $C$ also maintains the element $\left(I D_{S E, i}, F_{G, S E, i, 1}, F_{T, S E, i, 2}\right)$ in $L_{S K^{*}}$

- Public key retrieve query $\boldsymbol{Q}_{P K}\left(I D_{P K, i}\right)$ : When $A_{N L-I}$ issues the $i$-th Public key retrieve query along with an identity $I D_{P K, i} \in Z_{p}^{*}$, the challenger $C$ performs the following steps:

_ C checks whether $I D_{P K, i}$ has been recorded in the list $L_{I K}$. If so, $C$ obtains the polynomial of QID for $I D_{P K, i}$ in $L_{I K}$. Otherwise, $C$ performs the Initial Key extract query $\left(I D_{P K, i}\right)$ to set the polynomial of QID for $I D_{P K, i}$.
- C checks whether $I D_{P K, i}$ has been record in the list $L_{S K}$. If so, $C$ obtains the polynomial of $R I D$ for $I D_{P K, i}$ in $L_{S K}$. Otherwise, $C$ performs the Secret key extract query $\left(I D_{P K, i}\right)$ to set the polynomial of $R I D$ for $I D_{P K, i}$.

_ Finally, $C$ answers the query by two bit strings of QID and RID by searching the lists $L_{G}$ and $L_{T}$, respectively.

Public key replace query $\boldsymbol{Q}_{P R}\left(I D_{P R, i}, \xi_{T, P R, i, 2}\right)$ : By this query, a type I adversary $A_{N L \text {-I }}$ can replace the original partial public key RID of a user with identity $I D_{P R, I}$ by the bit string $\xi_{T, P R, i, 2}$. In other words, $A_{N L-I}$ can choose a valid SID and set the corresponding $R I D$ by herself/himself. $C$ must record this replacement. More precisely, $C$ first translates $\xi_{T, P R, i, 2}$ to the polynomial $F_{T, P R, i, 2}$ by searching the list $L_{T}$. Since $A_{N L-I}$ can generate valid user's secret key by using the group oracles, thus $C$ can obtain the polynomial $F_{G, P R, i, 1}$ by searching $F_{T, P R, i, 2}=F_{G, P R, i, 1} \cdot g$ in the list $L_{G}$. The challenger $C$ then update the user's secret key $\left(I D_{P R, i}, S I D_{P R, i}, R I D_{P R, i}\right)=\left(I D_{P R, i}, F_{G, P R, i, 1}, F_{T, P R, i, 2}\right)$ in the list $L_{S K}$.

Sign query $Q \boldsymbol{s}\left(I D_{S i}, m_{i}\right)$ : For the $i$-th Sign query, upon receiving this query along with an identity $I D_{S i} \in Z_{p}^{*}$ and a message $m_{i} \in Z_{p}^{*}$, the challenger $C$ returns $\left(\xi_{G, S, i, 1}, \xi_{G, S, i, 2}\right)$ to $A_{N L-\mathrm{I}}$ at the end of this query. When $C$ receives the query, $C$ respectively obtains the user's private key DID and secret key $S I D$ from the lists $L_{I K}$ and $L_{S K}$ by the following steps:

_ $C$ checks whether the user's private key of $I D_{S i}$ has been recorded in the list $L_{I K}$. If so, $C$ obtains DID of $I D_{S i}$ in $L_{I K}$. Otherwise, $C$ performs the query $Q_{I E}\left(I D_{S i}\right)$ to obtains $D I D$.

_ $C$ checks whether the user's secret key SID of $I D_{S i}$ has been recorded in the list $L_{S K}$. If so, $C$ obtains $S I D$ of $I D_{S i}$ in $L_{S K}$. Otherwise, $C$ performs the query $Q_{S E}\left(I D_{S i}\right)$ to obtain $S I D$.

- Hence, $C$ can obtain the polynomials $F_{G, I E, k, 1}$ and $F_{G, S E, l, 1}$ representing DID and SID, respectively.

Then $C$ can return $\left(\sigma_{1}, \sigma_{2}\right)=\left(\xi_{G, S, i, 1}, \xi_{G, S, i, 2}\right)$ to $A_{N L-\mathrm{I}}$ by running the following steps:

_ In order to generate $\sigma_{2}, C$ first defines a new variate $T_{G, S, i, 2}$ in $G$ and sets $F_{G, S, i, 2}=T_{G, S, i, 2}$. 
Moreover, $C$ selects a random bit string $\xi_{G, S, i, 2}$ which is distinct from all the $\xi_{G, \Theta, k, l}$ in $L_{G}$. Then $C$ adds $\left(F_{G, S, i, 2}, \xi_{G, S, i, 2}\right)$ in $L_{G}$ while computing the polynomial $F_{G, S, i, 1}=F_{G, I E, k, 2}+$ $F_{G, S E, l, 1}+\left(M_{0}+m_{j} M_{1}\right) \cdot T_{G, S, i, 2}$.

_ Finally, $C$ tries to find a pair $\left(F_{G, \omega, j, l}, \xi_{G, \omega, j, l}\right)$ in $L_{G}$ such that $F_{G, \omega, j, l}=F_{G, S, i, 1}$. If so, $C$ returns $\left(\xi_{G, \omega, j, l}\right.$, $\left.\xi_{G, S, i, 2}\right)$ to $A_{N L-\mathrm{I}}$. Otherwise, $C$ selects a random bit string $\xi_{G, S, i, 1}$ which is distinct from all the $\xi_{G, \omega, k, l}$ in $L_{G}$. Finally, $C$ adds $\left(F_{G, S, i, 1}, \xi_{G, S, i, 1}\right)$ in $L_{G}$ and returns $\left(\sigma_{1}, \sigma_{2}\right)=\left(\xi_{G, S, i, 1}, \xi_{G, S, i, 2}\right)$ to $A_{N L-\mathrm{I}}$.

- Forgery phase: In this phase, the adversary $A_{N L-\mathrm{I}}$ outputs a forgery signature $\left(m^{*}, I D^{*}, \sigma^{*}=\left(\xi_{G, f, i, 1}\right.\right.$, $\left.\xi_{G, f, i, 2}{ }^{*}\right)$, where there are two restrictions: (1) ID has never been issued during the Initial key extract query $Q_{I E}$; (2) $\left(m^{*}, I D^{*}\right)$ has never been issued during the Sign query $Q_{S}$.

In the following, before discussing the probability that $A_{N L-\mathrm{I}}$ wins the game $g_{N L-\text { I }}$, we define several restrictions and notations as below:

1 In the game $g_{N L-1}, A_{N L-\mathrm{I}}$ can issue eight kinds of queries $O_{G}, O_{T}, O_{P}, Q_{I E}, Q_{S E}, Q_{P K}, Q_{P R}$ and $Q_{S}$. Let $q_{O}$ denote the total number of three oracles $O_{G}, O_{T}$ and $O_{P}$ issued by $A_{N L-\mathrm{I}}$. In addition, let $q_{I E}, q_{S E}, q_{P K}, q_{P R}$ and $q_{S}$ respectively denote the numbers of the queries $Q_{I E}, Q_{S E}, Q_{P K}, Q_{P R}$ and $Q_{S}$ issued by $A_{N L-\mathrm{I}}$. Since $A_{N L \text {-I }}$ can issue queries at most $q$ times, we have $q \geqq q_{O}+q_{I E}+q_{S E}+q_{P K}+q_{P R}+q_{S}$. Moreover, we define several sets as follows.

- $\{S\}$ : The set of both variates $S_{G, O, i, j}$ defined in $O_{G}$ and $S_{G, P, i, j}$ defined in $O_{P}$.

- $\{V\}$ : The set of the variates $V_{T, O, i, j}$ defined in $O_{T}$.

- $\{T\}$ : The set of the variates $T_{G, I E, i, 2}$ defined in $Q_{I E}$, $T_{G, S, i, 3}$ defined in $Q s$ and $T_{G, S E, i, 1}$ defined in $Q_{S E}$.

_ $\left\{F_{G}\right\}$ : The set of the polynomials $F_{G, O, i, k}, F_{G, E E, i, k}$ and $F_{G, S, i, k}$ in the Queries phase.

_ $\left\{F_{T}\right\}$ : The set of the polynomials $F_{T, O, i, k}$ and $F_{T, P, i, k}$ in the Queries phase.

2 Let $\left|L_{G}\right|$ and $\left|L_{T}\right|$ denote the total numbers of tuples in the lists $L_{G}$ and $L_{T}$, respectively. Meanwhile, we have $\left|L_{G}\right|+\left|L_{T}\right| \leqq 3 q_{O}+2 q_{I E}+2 q_{S E}+4 q_{P K}+5 q_{S}+9 \leqq 5 q$ due to the following reasons:

In each query of $O_{G}, O_{T}$ and $O_{P}$, there are at most three elements involved in the query. So, the elements generated $O_{G}, O_{T}$ and $O_{P}$ is bounded by $3 q_{O}$, where $q_{O}$ denotes the total time of three oracles issued by $A_{N L-\mathrm{I}}$. In addition, no new elements of both $L_{G}$ and $L_{T}$ are generated in the query $Q_{P R}$.

_ For the query $Q_{I E}$, there are at most two new elements added in the list $L_{G}$. So, the increasing numbers of $\left|L_{G}\right|+\left|L_{T}\right|$ is bounded by $2 q_{I E}$.

- For the query $Q_{S E}$, there are at most two new elements added in the list $L_{G}$ or $L_{T}$. So, the increasing numbers of $\left|L_{G}\right|+\left|L_{T}\right|$ is bounded by $2 q_{S E}$.

_ For the query $Q_{P K}$, there are at most four new elements added in the list $L_{G}$ or $L_{T}$. So, the increasing numbers of $\left|L_{G}\right|+\left|L_{T}\right|$ is bounded by $4 q_{P K}$.

_ For the query $Q_{S}$, there are at most five new elements added in the list $L_{G}$ or $L_{T}$. So, the increasing numbers of $\left|L_{G}\right|+\left|L_{T}\right|$ is bounded by $6 q_{S}$.

Hence $\left|L_{G}\right|+\left|L_{T}\right| \leqq 7+3 q_{O}+2 q_{I E}+2 q_{S E}+4 q_{P K}+6 q_{S}+2$. Let $9 \leqq 3 q_{O}+4 q_{I E}+4 q_{S E}+2 q_{P K}+6 q_{P R}$, we have $\left|L_{G}\right|+\left|L_{T}\right| \leqq$ $3 q_{O}+2 q_{I E}+2 q_{S E}+4 q_{P K}+6 q_{S}+9 \leqq 6 q$.

3 The degrees of all multivariate polynomials in the set $\left\{F_{G}\right\}$ are at most 2 by the following reasons:

_ All the elements in $\{S\}$ and $\{T\}$ are polynomials with only one term, hence all the polynomials in $\{S\}$ and $\{T\}$ are of degree 1 .

_ For $Q_{I E}$, each polynomial $F_{G, I E, i, k}$ has degree at most 2 .

_ For $Q_{S E}$, each polynomial $F_{G, S E, i, 1}$ has degree 1.

_ For $Q_{S}$, each polynomial $F_{G, S, i, k}$ has degree at most 2.

- For $O_{G}$, the degree of $F_{G, 0, i, 1}+F_{G, 0, i, 2}$ is equal to the maximal degree of $F_{G, 0, i, 1}$ and $F_{G, 0, i, 2}$.

4 The degrees of all multivariate polynomials in the set $\left\{F_{T}\right\}$ are at most 4 by the following reasons:

_ All the elements in $\{V\}$ are polynomials with only one term, hence all the polynomials in $\{V\}$ are of degree 1.

_ For $O_{P}$, each polynomial $F_{T, P, i, k}$ has degree at most 4 since the degree of $F_{G}$ is at most 2 .

_ For $Q_{S E}$ each polynomial $F_{T, S E, i, 2}$ has degree 2.

- For $O_{T}$, the degrees of $F_{T, 0, i, 1}+F_{T, O, i, 2}$ are equal to the maximal degree of $F_{T, 0, i, 1}$ and $F_{T, 0, i, 2}$.

Assume that $A_{N L-I}$ generates a signature $\left(m^{*}, I D^{*}\right.$, 
$\left.\sigma^{*}=\left(\xi_{G, f, 1,1} * \xi_{G, f, 1,2}{ }^{*}\right)\right)$ while $I D^{*}$ is not issued in the query $Q_{I E}$ and $\left(m^{*}, I D^{*}\right)$ is not issued in the query $Q_{S^{*}} C$ first uses $I D^{*}$ to obtain the polynomials of QID and RID, denoted by $Q I D_{I D^{*}}$ and $R I D_{I D^{*}}$, respectively. Let $F_{G f, 1,3}$ and $F_{G f, 1,4}$ be the polynomials corresponding to $Q I D_{I D^{*}}$ and $R I D_{I D^{*}}$ respectively. Also, let $F_{G f, 1,1}$ and $F_{G f, 1,2}$ be the polynomials corresponding to $\xi_{G f, 1,1}{ }^{*}$ and $\xi_{G f, 1,2}{ }^{*}$,respectively. Then $C$ computes the polynomial $F_{G, f, 1,5}=X+$ $F_{G, f, 1,4}+\left(U_{0}+I D^{*} \cdot U_{1}\right) \cdot F_{G, f, 1,3}+\left(M_{0}+m^{*} M_{1}\right) \cdot F_{G, f, 1,2}-F_{G, f, 1,1}$. Note that the polynomial $F_{G, f, 1,5}$ has degree at most 3. Moreover, $C$ selects the random values $x, u_{0}, u_{1}, m_{0}, m_{1}$, $\left\{s_{1}, s_{2}, \ldots, s_{\mid\{S\}\}}\right\}$ and $\left\{t_{1}, t_{2}, \ldots, t_{\mid\{\mathrm{T}\}\}}\right\}$ in $Z_{p}^{*}$ and generates the corresponding values $X, U_{0}, U_{1}, M_{0}, M_{1},\{S\},\{T\}$ in the group $G$. $C$ also selects the random values $\left\{v_{1}, v_{2}, \ldots\right.$, $\left.v_{\{\{\mathrm{V}\} \mid}\right\}$ in $Z_{p}^{*}$ and generates $\{V\}$ in the group $G_{T}$.

Here, let us discuss the situations that $A_{N L-\mathrm{I}}$ wins the game $g_{N L-\mathrm{I}}$. We say that $A_{N L-\mathrm{I}}$ wins the game $\boldsymbol{g}_{N L-\mathrm{I}}$ if one of the following two cases occurs:

- Case 1. There exists a collision in group $G$ or $G_{T}$. We describe them as below:

- There are two polynomials $F_{G, i}$ and $F_{G, j}$ in the list $L_{G}$ such that $F_{G, i}\left(x, m_{0}, m_{1}, u_{0}, u_{1},\{s\},\{t\}\right)=$ $F_{G, j}\left(x, m_{0}, m_{1}, u_{0}, u_{1},\{s\},\{t\}\right)$.

- There are two polynomials $F_{T, i}$ and $F_{T, j}$ in the list $L_{T}$ such that $F_{T, i}\left(x, m_{0}, m_{1}, u_{0}, u_{1},\{s\},\{t\}\right.$, $\{v\})=F_{T, j}\left(x, m_{0}, m_{1}, u_{0}, u_{1},\{s\},\{t\},\{v\}\right)$.

- Case 2. In the forgery phase, the adversary $A_{N L-\mathrm{I}}$ generates the forgery signature $\left(m^{*}, I D^{*},\left(\xi_{G, f, i, 1}\right.\right.$, $\left.\left.\xi_{G_{f, j, 2}}{ }^{*}\right)\right)$ which satisfies the equality $F_{G, f, 5}\left(x, m_{0}, m_{1}\right.$, $\left.u_{0}, u_{1},\{s\},\{t\}\right)=0$, where $F_{G, f, 1,5}$ is computed earlier.

In the real UF-CLS-ACMA game defined in Definition 1 , the success probability in the game $g_{N L-\mathrm{I}}$ is an upper bound of the advantage of $A_{N L-\mathrm{I}}$. In the following, we discuss the probabilities of two cases in the game $g_{N L-\mathrm{I}}$. The probabilities of two cases are computed as below:

- Case 1. If there exists a collision in group $G$ or $G_{T}$, then one may solve the discrete logarithm problem in $G$ or $G_{T}$ [26]. Assume that $F_{G, i}$ and $F_{G, j}$ denote two distinct polynomials in $L_{G}$ such that $F_{G, i}\left(x, m_{0}, m_{1}\right.$, $\left.u_{0}, u_{1},\{s\},\{t\}\right)=F_{G, j}\left(x, m_{0}, m_{1}, u_{0}, u_{1},\{s\},\{t\}\right)$. In such a case, the polynomial $F_{G, C}=F_{G, i}-F_{G, j}$ is a non-zero polynomial, whose degree is at most 2. By Lemma 2 in Section 2, the probability of $F_{G, C}\left(x, m_{0}, m_{1}, u_{0}, u_{1}\right.$, $\{s\},\{t\})=0$ in $Z_{p}$ is at most $2 / p$. Since $\left|L_{G}\right|$ denotes the total number of tuples in the list $L_{G}$, there are $\left(\begin{array}{c}\left|L_{G}\right| \\ 2\end{array}\right)$ possible pairs $\left(F_{G, i}, F_{G, j}\right)$. The collision probability in $L_{G}$ is at most $(2 / p)\left(\begin{array}{l}\left.\mid \begin{array}{l}L_{G} \\ 2\end{array}\right) \\ )\end{array}\right)$ Similarly, since the maximal degree of polynomials in $L_{T}$ is at most 4 , the collision probability in $L_{T}$ is at most $(4 / p)\left(\begin{array}{l}\left|L_{T}\right| \\ 2\end{array}\right)$.

- Case 2. In this case, the success probability of $A_{N L-\mathrm{I}}$ is the probability that $A_{N L-\mathrm{I}}$ can forge a valid signature $\left(m^{*}, I D^{*}, \quad \sigma^{*}=\left(\xi_{G, f, i, 1}^{*}, \quad \xi_{G, f, i, 2}{ }^{*}\right)\right)$ which satisfies the equality $F_{G, f, 1,5}\left(x, m_{0}, m_{1}, u_{0}, u_{1},\{s\},\{t\}\right)=$ 0 , where $F_{G, f, 1,5}=X+F_{G, f, 1,4}+\left(U_{0}+I D^{*} \cdot U_{1}\right) \cdot F_{G, f, 1,3}+\left(M_{0}+\right.$ $\left.m^{*} M_{1}\right) \cdot F_{G f, 1,2}-F_{G f, 1,1}$. Here, the polynomial $F_{G f, 1,5}$ has degree at most 3. In the meantime, $F_{G, f, 1,5}$ is a nonzero polynomial that will be proved in Lemma 3 later. In such a case, by Lemma 2 in Section 2 , the probability of Case 2 is at most $3 / p$.

Since $\left|L_{G}\right|+\left|L_{T}\right| \leqq 6 q$ as mentioned earlier, the advantage that $A_{N L-\mathrm{I}}$ wins the game $g_{N L-\mathrm{I}}$ in Case 1 or 2 is at most

$$
\begin{aligned}
& (2 / p)\left(\begin{array}{l}
\left|L_{G}\right| \\
2
\end{array}\right)+(4 / p)\left(\begin{array}{c}
\left|L_{T}\right| \\
2
\end{array}\right)+ \\
& (3 / p) \leqq(2 / p)\left(\left|L_{G}\right|+\left|L_{T}\right|\right)^{2} \leqq 72 q^{2} / p \text {, }
\end{aligned}
$$

which is negligible if $q=p o l y(\log p)$.

Lemma 3. The polynomial $F_{G f, 1,5}=X+F_{G f, 1,4}+\left(U_{0}+\right.$ $\left.I D^{*} \cdot U_{1}\right) \cdot F_{G f, 1,3}+\left(M_{0}+m^{*} M_{1}\right) \cdot F_{G f, 1,2}-F_{G f, 1,1}$ is a non-zero polynomial.

Proof: By the group oracle $O_{G}$ in the game $g_{N L-\text { I }}$, the increased elements (polynomials) in $L_{G}$ are obtained by adding or subtracting two polynomials in $L_{G}$. In such a case, we may write $F_{G f, 1, b}$ for $l=1,2,3,4$, as the following form,

$$
\begin{aligned}
& F_{G, f, 1, l}=c_{l, 1}+c_{l, 2} U_{1}+c_{l, 3} U_{0}+c_{l, 4} M_{0} \\
& +c_{l, 5} M_{1}+\sum_{i=1}^{3 q_{g}}\left(d_{l, 6, i} \cdot S_{i}\right)+\sum_{i=1}^{q_{S}+q_{I E}}\left(d_{l, 7, i} \cdot T_{i}\right) \\
& +\sum_{i=1}^{q_{I E}}\left(d_{l, 8, i} \cdot D I D_{I E, i}\right)+\sum_{j=1}^{q_{S E}}\left(d_{l, 9, j} \cdot S_{S E, j}\right) \\
& +\sum_{i=1}^{q_{S}}\left(d_{l, 10, k}\left(D I D_{I E, i}+S I D_{S E, k}+\left(M_{0}+m_{k} \cdot M_{1}\right) \cdot T_{G, S, k, 2}\right)\right),
\end{aligned}
$$

where $D I D_{I E, i}=X+\left(U_{0}+I D_{I E, i} \cdot U_{1}\right) \cdot T_{G, I E, i, 2}$ for $1 \leqq i \leqq q_{I E}$, and $S I D_{S E, j}=T_{G, S E, j, 1}$ for $1 \leqq j \leqq q_{S E}$. In addition, $S_{i}$ and $T_{i}$ respectively run through all the elements in the sets $\{S\}$ and $\{T\}$. It is worth mentioning, that each $c_{i j}$ and $d_{i, j, k}$ in $Z_{p}$ are randomly selected by the adversary $A_{N L-\mathrm{I}}$. In the following, we discuss three cases to show that $F_{G, f, 1,5}$ is a non-zero polynomial.

1 Case 1. If $\sum_{j=1}^{q_{I E}} d_{2,8, j}=\sum_{j=1}^{q_{I E}} d_{3,8, j}=\left(\sum_{j=1}^{q_{I E}} d_{4,8, j}-\right.$ 
$\left.\sum_{j=1}^{q_{I E}} d_{1,8, j}\right)=0$ and $d_{2,10, k}=d_{3,10, k}=\left(d_{4,10, k}-d_{1,10, k}\right)=0$ for all $1 \leqq j \leqq q_{I E}$ and $1 \leqq k \leqq q_{S}$, then $F_{G_{f f, 1,2}}, F_{G, f, 1,3}$ and $\left(F_{G f, 1,4}-F_{G, f, 1,1}\right)$ do not contain the indeterminate $X$. In such a case, the coefficient of the term $X$ in $F_{G, f, 1,5}$ is 1 . Therefore, $F_{G f, 1,5}$ must be non-zero.

2 Case 2: At least one of $d_{2,10, k}, d_{3,10, k}$ and $\left(d_{4,10, k}-d_{1,10, k}\right)$ is non-zero for some $k$.

_ If $d_{2,10, k} \neq 0$ for some $k, F_{G, f, 1,5}$ is non-zero since $d_{2,10, k}$ is the coefficient of the term $M_{0}^{2} \cdot T_{G, S, k, 2}$ in $F_{G, f, 1,5}$.

- If $d_{3,10, k} \neq 0$ for some $k, F_{G, f, 1,5}$ is non-zero since $d_{3,10, k}$ is the coefficient of the term $U_{0} \cdot M_{0} \cdot T_{G, S, k, 2}$ in $F_{G, f, 1,5}$.

_ If $\left(d_{4,10, k}-d_{1,10, k}\right) \neq 0$ for some $k$, we discuss the following two cases.

_ If $\left(\sum_{i=1}^{q_{S}+q_{I E}} d_{2,7, i}\right)+\left(d_{4,10, k}-d_{1,10, k}\right) \neq 0$, then $F_{G, f, 1,5}$ is non-zero since $\left(\sum_{i=1}^{q_{S}+q_{I E}} d_{2,7, i}\right)+\left(d_{4,10, k}-\right.$ $\left.d_{1,10, k}\right)=d_{4,9, k}+\left(\sum_{i=1}^{q_{S}+q_{I E}} d_{2,7, i}\right)-d_{1,9, k}$ is the coefficient of the term $M_{0} \cdot T_{G, S, k, 2,2}$ in $F_{G, f, 1,5}$.

_ If $\left(\sum_{i=1}^{q_{S}+q_{I E}} d_{2,7, i}\right)+\left(d_{4,10, k}-d_{1,10, k}\right)=0$, then $\left(\sum_{i=1}^{q_{S}+q_{I E}} d_{2,7, i}\right)=-\left(d_{4,10, k}-d_{1,10, k}\right) \neq 0$. And, in this case, the coefficient of the term $M_{1} \cdot T_{G, S, k, 2}$ in $F_{G, f, 1,5}$ is $m^{*} \cdot\left(\sum_{i=1}^{q_{S}+q_{I E}} d_{2,7, i}\right)+\left(d_{4,10, k}-d_{1,10, k}\right)$. Without loss of generality, letting $m^{*} \neq-1$, we have $F_{G f, 1,5}$ is non-zero .

3 Case 3: Otherwise, under the condition $d_{2,10, k}=$ $d_{3,10, k}=\left(d_{4,10, k}-d_{1,10, k}\right)=0$ for all $1 \leqq k \leqq q_{S}$, the terms $\sum_{i=1}^{q_{S}}\left(d_{l, 10, k}\left(D I D_{I E, i}+S I D_{S E, k}+\left(M_{0}+m_{k} \cdot M_{1}\right) \cdot T_{G, S, k, 2}\right)\right)$ in $F_{G, f, 1, l}$, for $l=1,2,3,4$, no longer affect $F_{G, f, 1,5}$. In such a case, the polynomial $F_{G, f, l, l}$ can be simplified to

$F_{G, f, 1, l}=c_{l, 1}+c_{l, 2} U_{1}+c_{l, 3} U_{0}+c_{l, 4} M_{0}+c_{l, 5} M_{1}$

$+\left(d_{l, 6, i} \cdot S_{i}\right)+\sum_{i=1}^{q_{S}+q_{I E}}\left(d_{l, 7, i} \cdot T_{i}\right)$

$+\sum_{i=1}^{q_{I E}}\left(d_{l, 8, i} \cdot D I D_{I E, i}\right)+\sum_{j=1}^{q_{S E}}\left(d_{l, 9, j} \cdot \operatorname{SID}_{S E, j}\right)$,

where $D I D_{I E, i}=X+\left(U_{0}+I D_{I E, i} \cdot U_{1}\right) \cdot T_{G, E E, i, 2}$ for $1 \leqq i \leqq q_{I E}$. We discuss the following three cases:

- If $\sum_{i=1}^{q_{I E}} d_{3,8, i} \neq 0$, then $d_{3,8, i} \neq 0$ for some $i$. Hence, $F_{G, f, 1,5}$ is non-zero since $d_{3,8, i}$ is the coefficient of the term $U_{0}^{2} \cdot T_{G, I E, j, 2}$ in $F_{G f, 1,5}$.

- If $\sum_{i=1}^{q_{I E}} d_{2,8, i} \neq 0$, then at least one $d_{2,8, i}$ is non-zero for some $i$. Hence, the coefficient of the term $M_{0} \cdot U_{0} \cdot T_{G, I E, i, 2}$ in $F_{G, f, 1,5}$ is non-zero and so $F_{G f, 1,5}$ is non-zero .

- If $\left(\sum_{i=1}^{q_{I E}} d_{4,8, j}-\sum_{i=1}^{q_{I E}} d_{1,8, j}\right) \neq 0$, then at least one $\left(d_{4,8, j}-d_{1,8, j}\right)$ is non-zero for some $j$. If $d_{3,7, i}+\left(d_{4,8, j}\right.$ $\left.d_{1,8, j}\right) \neq 0$, then $F_{G, f, 1,5}$ is non-zero since $d_{3,7, i}+\left(d_{4,8, j}\right.$ $\left.d_{1,8, j}\right)$ is the coefficient of the term $U_{0} \cdot T_{G, I E, i, 2}$ in $F_{G f, 1,5^{\circ}}$ Otherwise, $d_{3,7, i}=d_{1,8, j}-d_{4,8, j} \neq 0$. In this case, the coefficient of the term $U_{1} \cdot T_{G, E E, j, 2}$ in $F_{G, f, 1,5}$ is $I D^{*} \cdot d_{3,7, i}-\left(d_{1,8, j}-d_{4,8, j}\right) \cdot I D_{I E, j}=\left(d_{1,8, j}-d_{4,8, j}\right) \cdot\left(I D^{*}\right.$ $\left.I D_{I E, j}\right)$ which is non-zero since $I D^{*} \neq I D_{I E, j}$ for $1 \leqq j \leqq q_{I E}$.

Theorem 2. In the generic bilinear group model, the non-leakage version $\Pi_{N L}$ of the proposed LR-CLS scheme is provably secure against the Type II adversary (honest-but-curious KGC).

Proof: Let $A_{N L-I I}$ be a Type II adversary who can break the non-leakage CLS scheme $\Pi_{N L}$ while $A_{N L \text {-II }}$ is allowed to issue all the queries at most $q$ times. The advantage of $A_{N L \text {-II }}$ is defined as the probability that $A_{N L \text {-II }}$ wins the following game $g_{N L-I I}$ played with a challenger $C$.

Game $\boldsymbol{g}_{N L-I I}$ In the game $g_{N L-I I}$, there are three phases, namely, Setup, Queries and Forgery phases. At the end of this game, $A_{N L-\text { II }}$ outputs a forgery signature. Three phases are described as below:

- Setup phase: In this phase, the challenger $C$ prepares two initial-empty lists $L_{G}$ and $L_{T}$ to record the tuples in $G$ and $G_{T}$, respectively. The forms of $L_{G}$ and $L_{T}$ are the same with those described in the game $g_{N L-\mathrm{I}}$. The challenger $C$ also maintains two lists $L_{I K}$ and $L_{S K}$ to record the tuples of users' initial keys and secret keys, respectively. At the end of this phase, $C$ sends the bit strings of the public parameters to $A_{N L-I I}$. Since the type II adversary $A_{N L-I I}$ models an honest-but-curious KGC, $C$ sends the bit string of the system secret key $X$ along with the public parameters to $A_{N L-I I}$.

Queries phase: Since $A_{N L \text {-II }}$ models an honest-butcurious KGC, $A_{N L-I I}$ can compute the user's initial key by issuing the oracles $O_{G}, O_{T}$ and $O_{E}$. Meanwhile, $A_{N L \text {-II }}$ cannot perform the public key replacement query in this game. Hence in this phase, $A_{N L \text {-II }}$ can issue six kinds of queries as below:

- Group oracle $O_{G}\left(\xi_{G, 0, i, 1}, \xi_{G, O, i, 2}\right.$, operation): This query is identical to $O_{G}$ described in $g_{N L-\mathrm{I}}$. 
- Group oracle $\boldsymbol{O}_{T}\left(\xi_{T, O, i, 1}, \xi_{T, O, i, 2}\right.$, operation): This query is identical to $O_{T}$ described in $g_{N L-\mathrm{I}}$.

_ Pairing oracle $O_{P}\left(\xi_{G, P, i, 1}, \xi_{G, P, i, 2}\right)$ : This query is identical to $O_{P}$ described in $g_{N L-\mathrm{I}}$.

_ Secret key extract query $Q_{S E}\left(I D_{S E, i}\right)$ : This query is identical to $Q_{S E}$ described in $g_{N L-\mathrm{I}}$.

- Public key retrieve query $\boldsymbol{Q}_{P K}\left(I D_{P K, i}\right)$ : When $A_{N L-I I}$ issues the $i$-th Public key retrieve query along with an identity $I D_{P K, i} \in Z_{p}^{*}$, the challenger $C$ performs the following three steps:

_ C checks whether $I D_{P K, i}$ has been recorded in the list $L_{I K}$. If so, $C$ obtains the polynomial of QID for $I D_{P K, i}$ in $L_{I K}$. Otherwise, $C$ checks the records of the oracles $O_{G}, O_{T}$ and $O_{E}$ to obtain the polynomials of (DID, QID) for $I D_{P K, i}$ and update the list $L_{I K}$ for $I D_{P K, i}$.

_ $C$ checks whether $I D_{P K, i}$ has been recorded in the list $L_{S K}$. If so, $C$ obtains the polynomial of $R I D$ for $I D_{P K, i}$ in $L_{S K}$. Otherwise, $C$ performs the Secret key extract query $\left(I D_{P K, i}\right)$ to set the polynomial of $R I D$ for $I D_{P K, i}$.

_ Finally, $C$ answers the query by two bit strings of QID and RID by searching the lists $L_{G}$ and $L_{T}$, respectively.

Sign query $\mathbf{Q}_{S}\left(I D_{S i}, m_{i}\right)$ : For the $i$-th Sign query, upon receiving this query along with an identity $I D_{S i} \in Z_{p}^{*}$ and a message $m_{i} \in Z_{p}^{*}$, the challenger $C$ returns $\left(\xi_{G, S, i, 1}, \xi_{G, S, i, 2}\right)$ to $A_{N L \text {-II }}$ at the end of this query. When $C$ receives the query, $C$ respectively obtains the user's private key DID and secret key SID from the lists $L_{I K}$ and $L_{S K}$ by the following steps:

- $C$ checks whether the user's private key of $I D_{S i}$ has been recorded in the list $L_{I K}$. If so, $C$ obtains $D I D$ of $I D_{S i}$ in $L_{I K}$. Otherwise, $C$ first checks the record of the oracles $O_{G}, O_{T}$ and $O_{E}$ to obtain the polynomials of (DID, QID) for $I D_{S i}$ and then update the list $L_{I K}$ for $I D_{S, i}$.

- $C$ checks whether the user's secret key SID of $I D_{S i}$ has been recorded in the list $L_{S K}$. If so, $C$ obtains $S I D$ of $I D_{S i}$ in $L_{S K}$. Otherwise, $C$ performs the query $Q_{S E}\left(I D_{S i}\right)$ to obtain the tuple $S I D$ of $I D_{S i}$.

- Hence, $C$ can obtain the polynomials $F_{G, I E, k, 1}$ and $F_{G, S E, l 1}$ representing $D I D$ and SID, respectively.
The rest steps are identical to $Q_{S}$ described in the game $g_{N L-\mathrm{I}}$. Finally, $C$ generates and returns $\left(\sigma_{1}, \sigma_{2}\right)=\left(\xi_{G, S, i, 1}\right.$, $\left.\xi_{G, S, i, 2}\right)$ to $A_{N L-I \Gamma}$.

_ Forgery phase: In this phase, the type II adversary $A_{N L \text {-II }}$ outputs a forgery signature $\left(m^{*}, I D^{*}, \sigma^{*}=\right.$ $\left.\left(\xi_{G, f, 1,1} * \xi_{G, f, 2,2}\right)\right)$. It is worth mentioning, where there are two restrictions: (1) ID* has never been issued during the Secret key extract query $Q_{S E}$; (2) $\left(m^{*}, I D^{*}\right)$ has never been issued during the Sign query $Q s$.

In the real UF-CLS-ACMA game defined in Definition 1, the success probability in the game $g_{N L \text {-II }}$ is an upper bound of the advantage of $A_{N L \text {-II }}$. As the same arguments in Theorem 1 , we can compute the success probability of $A_{N L \text {-II }}$ in game $g_{N L-I I}$. By applying the same steps in Theorem 1 We have $\left|L_{G}\right|+\left|L_{T}\right| \leqq 7+3 q_{O}+2 q_{S E}$ $+4 q_{P K}+4 q_{S}+2$. Let $9 \leqq q_{O}+2 q_{S E}$, we have $\left|L_{G}\right|+\left|L_{T}\right| \leqq 3 q_{O}$ $+2 q_{S E}+4 q_{P K}+3 q_{S}+9 \leqq 4 q$. Now we can compute the success probability of $A_{N L-\text { II }}$ in game $g_{N L-I I}$. The advantage that $A_{N L \text {-II }}$ wins the game $g_{N L \text {-II }}$ is at most

$$
\begin{aligned}
& (2 / p)\left(\begin{array}{l}
\left|L_{G}\right| \\
2
\end{array}\right)+(4 / p)\left(\begin{array}{c}
\left|L_{T}\right| \\
2
\end{array}\right) \\
& +(3 / p) \leqq(2 / p)\left(\left|L_{G}\right|+\left|L_{T}\right|\right)^{2} \leqq 32 q^{2} / p,
\end{aligned}
$$

which is negligible if $q=p o l y(\log p)$.

In Theorems 1 and 2, we have proved the security of the non-leakage version of the proposed LR-CLS scheme. In the following, based on the security of the non-leakage version, we prove that the proposed LRCLS scheme under the continual leakage model is UFLR-CLS-ACMA secure against Type I and Type II adversaries in Theorems 3 and 4, respectively. Figure 5 demonstrates the conceptual principle of the security games $g_{L R-\mathrm{I}}$ and $g_{L R-\mathrm{II}}$ employed in Theorems 3 and 4 .

Theorem 3. In the generic bilinear group model, the proposed LR-CLS scheme is provably secure against the Type I adversary (outsider) under the continual leakage model.

Proof: We have proven that the non-leakage version of our proposed scheme is secure against the Type I adversary in Theorem 1 . Here, the adversary is allowed to issue two extra queries, namely, Initial key extract leak query and Sign leak query. Hence we modify the game described in Theorem 1. Let $A_{L R-\mathrm{I}}$ be a Type I adversary who can break our LR-CLS scheme $\Pi_{L R}$ while $A_{L R-\mathrm{I}}$ is allowed to issue all the queries at most $q$ times. The advantage of $A_{L R-\mathrm{I}}$ is defined as the probability 
Figure 5

The conceptual principle of the security games $g_{L R-\mathrm{I}}$ and $g_{L R-\text { II }}$ in Theorems 3 and 4

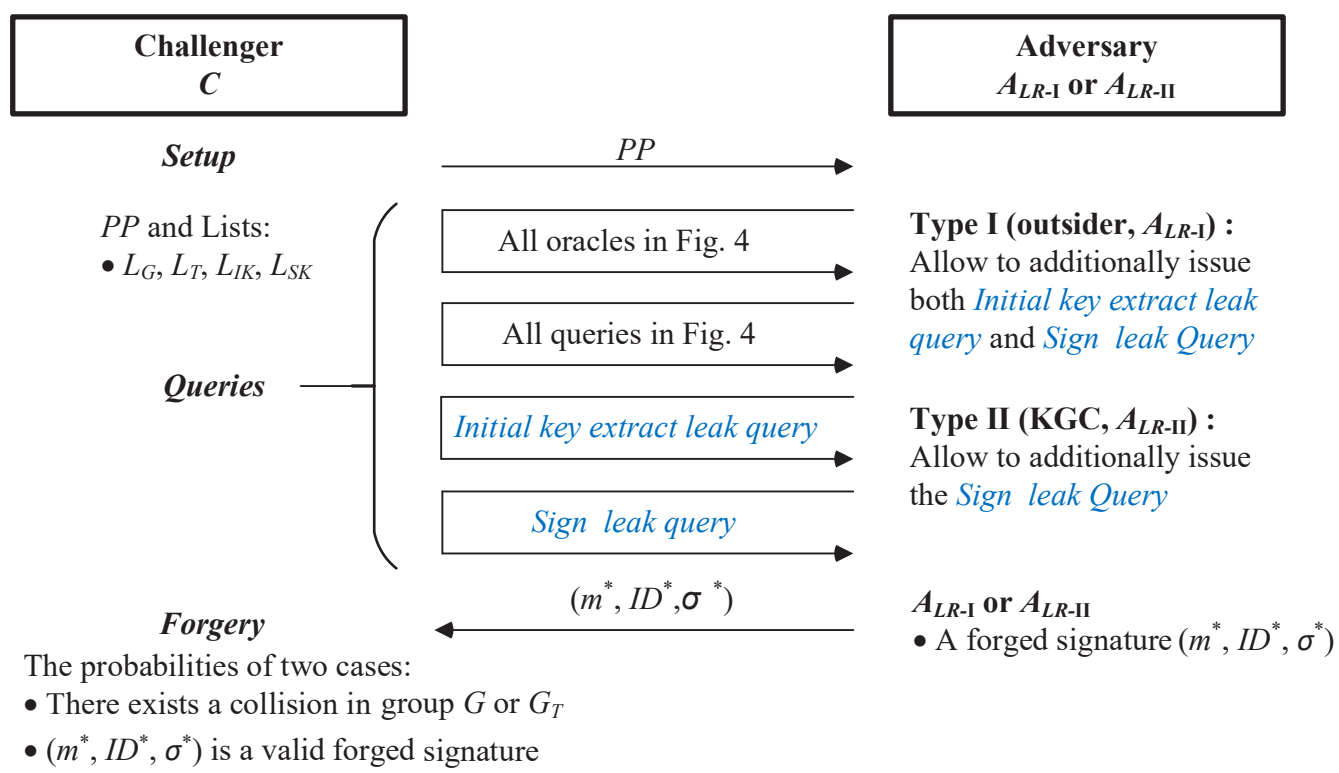

that $A_{L R-\mathrm{I}}$ wins the following game $g_{L R-\mathrm{I}}$ played with a challenger $C$.

Game $g_{L R-\mathrm{I}}:$ In the game $g_{L R-\mathrm{I}}$, there are three phases, namely, Setup, Queries and Forgery phases. At the end of this game, $A_{L R-\mathrm{I}}$ outputs a forgery signature. In Queries phase, $A_{L R \text {-I }}$ may issue ten kinds of queries in any order at most $q$ times. Three phases are described as below:

_ Setup phase: This phase is identical to that of the game $g_{N L-\mathrm{I}}$.

- Queries phase: In addition to the eight kinds of queries in the game $g_{N L-\mathrm{I}}, A_{L R-I}$ may issue two extra leakage queries (Initial key extract leak query and Sign leak query). In order to represent the leakage information, two leakage functions $f_{I E, i}$ and $h_{I E, i}$ model the ability of the adversary for Extract-1 and Extract-2 of the $i$-th Initial key extract round, respectively. Also, two leakage functions $f_{S, j}$ and $h_{S, j}$ are used to model the ability of the adversary for Sign-1 and Sign-2 of a user's $j$-th Sign round. Note that four leakage functions $f_{I E, i}, h_{I E, i}, f_{S, j}$ and $h_{S, j}$ respectively generate the leakage information $\Lambda f_{I E, i}$, $\Lambda h_{I E, i}, \Lambda f_{S, j}$ and $\Lambda h_{S, j}$. Meanwhile, four initial-empty lists $L_{f, I E}, L_{h, I E}, L_{f, S}$ and $L_{h, S}$ are used to record the related leakage functions and leakage information as follows:

$L_{f, I E}=\left\{\left(f_{I E, i}, \Lambda f_{I E, i}\right), 1 \leqq i \leqq q_{I E}\right\}$,
$L_{h, I E}=\left\{\left(h_{I E, i}, \Lambda h_{I E, i}\right), 1 \leqq i \leqq q_{I E}\right\}$,
$L_{f, S}=\left\{\left(f_{S, j}, \Lambda f_{S, j}\right), 1 \leqq j \leqq q_{S}\right\}$,
$L_{h, S}=\left\{\left(h_{S, j}, \Lambda h_{S, j}\right), 1 \leqq j \leqq q_{S}\right\}$.

In addition, we describe two extra leakage queries as below:

Initial key extract leak query $\mathbf{Q}_{I E-L}\left(f_{I E, i}, h_{I E, i}, i\right)$ : For the $i$-th Initial key extract leak query, upon receiving this query along with two leakage functions $f_{I E, i}$ and $h_{I E, I}$ such that $\left|f_{I E, i}\right| \leq \lambda$ and $\left|h_{I E, i}\right| \leq \lambda, C$ generates the leakage information $\Lambda f_{I E, i}=f_{I E, i}\left(S_{i-1,1}, \gamma_{i}, a_{i}\right)$ and $\Lambda h_{I E, i}=h_{I E, i}\left(S_{i-1,2}, T I_{I E}\right.$, $\left.a_{i}\right)$ and returns them to $A_{L R-\mathrm{I}}$. Meanwhile, $C$ adds $\left(f_{I E, i}, \Lambda f_{I E, i}\right)$ and $\left(h_{I E, i}, \Lambda h_{I E, i}\right)$ in the lists $L_{f, I E}$ and $L_{h, I E}$, respectively. It is worth mentioning, that $A_{L R-\mathrm{I}}$ can ask the $\boldsymbol{Q}_{I E-L}$ for the same identity only once.

_ Sign leak query $\mathbf{Q}_{S-L}\left(f_{S, j}, h_{S, j}, j\right)$ : For the $j$-th Sign query, upon receiving this query along with two leakage functions $f_{S, j}$ and $h_{S, j}$ such that $\left|f_{S, j}\right| \leq \lambda$ 
and $\left|h_{S, j}\right| \leq \lambda, C$ generates the leakage information $\Lambda f_{S, j}=f_{S, j}\left(D I D_{j-1,1}, \eta_{j}, b_{j}, c_{j}\right)$ and $\Lambda h_{S, j}=h_{S, j}\left(D I D_{j-1,2}\right.$, $\left.T I_{S}, b_{j}, c_{j}\right)$ and returns them to $A_{L R-\mathrm{I}}$. Meanwhile, $C$ adds $\left(f_{S, j}, \Lambda f_{S, j}\right)$ in $L_{f, S}$ and $\left(h_{S, j}, \Lambda h_{S, j}\right)$ in $L_{h, S}$.

- Forgery phase: In this phase, the type I adversary $A_{L R-\mathrm{I}}$ outputs a forgery signature $\left(m^{*}, I D^{*}, \sigma^{*}=\left(\xi_{G, f, i, 1}\right.\right.$, $\left.\xi_{G, f, i, 2}{ }^{*}\right)$ ). It is worth mentioning, where there are two restrictions: (1) $I D^{*}$ has never been issued during the Initial key extract query $Q_{I E}$; (2) $\left(m^{*}, I D^{*}\right)$ has never been issued during the Sign query $Q s$.

By making use of the leakage functions, it is obvious that the success probability (advantage) of $A_{L R-\mathrm{I}}$ in $g_{L R-\mathrm{I}}$ is higher than that of $A_{N L-\mathrm{I}}$ in the game $g_{N L-\mathrm{I}}$. For the Initial key extract leak query with two leakage functions $f_{I E, I}$ and $h_{I E, i}$, the adversary $A_{L R-\mathrm{I}}$ can obtain partial information of $\left(S_{i-1,1}, \gamma_{i}, a_{i}\right)$ and $\left(S_{i-1,2}, T I_{I E}, a_{i}\right)$ by the leakage information $\Lambda f_{I E, i}$ and $\Lambda h_{I E, \text {, }}$, respectively, as follows.

- $\gamma_{i}$ : The random value $\gamma_{i}$ is involved in the computation of the Initial key extract query to generate the initial key of the user with identity $I D_{I E, i}$. If $A_{L R-\mathrm{I}}$ has issued the Initial key extract query on $I D_{I E, i,}$, any forgery signature for $I D_{I E, i}$ is not accepted in the Forgery phase. In such a case, the leakage of $\gamma_{i}$ is useless to generate a signature for $A_{L R-\mathrm{I}}$ in the Forgery phase.

_ $\left(S_{i-1,1}, S_{i-1,2}\right)$ : The partial information of $\left(S_{i-1,1}, S_{i-1,2}\right)$ could help $A_{L R-\mathrm{I}}$ to learn the partial information of the system secret key $X$. So, $A_{L R-\mathrm{I}}$ can get at most $2 \lambda$ bits information of $X$.

_ $a_{i}$ : The random value $a_{i}$ is involved in the computation of the current system secret key $\left(S_{i, 1}\right.$, $S_{i, 2}$ ), but it is independent to the system secret key $X$. In such a case, $A_{L R-\mathrm{I}}$ can learn at most $\lambda$ bits of $S_{i, 1}$ and $S_{i, 2}$, respectively.

- $T I_{I E}$ : The temporary information $T I_{I E}$ is useless to obtain the user's initial private key. In addition to that, it is also useless to generate a signature for $A_{L R-\mathrm{I}}$ in the Forgery phase.

On the other hand, for the Sign leak query with two leakage functions $f_{S, j}$ and $h_{S, j}$, the adversary $A_{L R-\mathrm{I}}$ can obtain partial information of $\left(D I D_{j-1,1}, \eta_{j}, b_{j}, c_{j}\right)$ and $\left(D I D_{j-1,2}, T I_{S}, b_{j}, c_{j}\right)$ by the leakage information $\Lambda f_{S, j}$ and $\Lambda h_{S, j}$, respectively, as follows.

- $\eta_{j}$ : The random value $\eta_{j}$ is involved in the computation of generating a signature on $\left(I D_{S, j}\right.$, $\left.m_{j}\right)$. If $A_{L R-\mathrm{I}}$ has issued the Sign query on $\left(I D_{S, j}, m_{j}\right)$, any forgery signature for $\left(I D_{S, j}, m_{j}\right)$ is not accepted in the Forgery phase. In such a case, the leakage of $\eta_{j}$ is useless to generate a signature for $A_{L R-\mathrm{I}}$ in the Forgery phase.

_ $\left(D I D_{j-1,1}, D I D_{j-1,2}\right)$ :Thepartialinformation of $\left(D I D_{j-1,1}\right.$, $D I D_{j-1,2}$ ) could help $A_{L R-\mathrm{I}}$ to learn the partial information of the user's first initial key $D I D_{0}$. So, $A_{L R-\mathrm{I}}$ can get at most $2 \lambda$ bits information of $D I D_{0}$.

_ $b_{j}$ : The random value $b_{j}$ is involved in the computation of generating the user's initial key $\left(D I D_{j, 1}, D I D_{j, 2}\right)$. In such a case, $A_{L R-\mathrm{I}}$ learns at most $\lambda$ bits information about $D I D_{j, 1}$ and $D I D_{j, 2}$, respectively.

_ $c_{j}$ :Therandomvalue $c_{j}$ isinvolvedinthecomputation of generating the user's secret key $\left(S I D_{j, 1}, S I D_{j, 2}\right)$. In such a case, $A_{L R-\mathrm{I}}$ learns at most $\lambda$ bits information about $S I D_{j, 1}$ and $S I D_{j, 2}$, respectively.

_ $T I_{S}$ : The temporary information $T I_{S}$ is useless to generate a signature for $A_{L R-\mathrm{I}}$ in the Forgery phase.

Here, we evaluate the probability that $A_{L R-\mathrm{I}}$ wins the game $g_{L R-\mathrm{I}}$, denoted by $\operatorname{Pr}_{L R-\mathrm{I}}$. Note that since the type I adversary $A_{L R-\mathrm{I}}$ can obtain the secret key of any entity, $A_{L R-\mathrm{I}}$ can forge a valid signature whenever she/he obtains the system secret key $X$ or the target user's initial key $D I D_{0}$. In the following, we define three events of $\operatorname{Pr}_{L R-\mathrm{I}}$, namely, SSK, UIK and VFS.

1 The event $S S K$ denotes that $A_{L R-\mathrm{I}}$ can get the system secret key $X$ completely by two leakage functions $f_{I E, i}$ and $h_{I E, I}$.

2 The event $U I K$ denotes that $A_{L R-I}$ can get the target user's initial key $D I D_{0}$ completely by two leakage functions $f_{S, j}$ and $h_{S, j}$.

3 The event VFS denotes that $A_{L R-\mathrm{I}}$ can generate a valid forgery signature.

Meanwhile, we denote the events $\overline{S S K}$ and $\overline{U I K}$ are the complement events of SSK and UIK respectively. Therefore, the probability that $A_{L R-\mathrm{I}}$ wins the game $g_{L R-\mathrm{I}}$ is bounded by

$$
\begin{aligned}
& \operatorname{Pr}_{L R-\mathrm{I}}=\operatorname{Pr}[V F S] \\
&=\operatorname{Pr}[V F S \wedge S S K]+\operatorname{Pr}[V F S \wedge \overline{S S K}] \\
&=\operatorname{Pr}[V F S \wedge S S K]+\operatorname{Pr}[V F S \wedge \overline{S S K} \wedge U I K] \\
&+\operatorname{Pr}[V F S \wedge \overline{S S K} \wedge \overline{U I K}] \\
&= \operatorname{Pr}[V F S \wedge S S K]+\operatorname{Pr}[V F S \wedge \overline{S S K} \wedge \overline{U I K}] \\
&+\operatorname{Pr}[V F S \mid \overline{S S K} \wedge \overline{U I K}] \cdot \operatorname{Pr}[\overline{S S K} \wedge \overline{U I K}] .
\end{aligned}
$$


Since $\operatorname{Pr}[V F S \wedge S S K] \leqq \operatorname{Pr}[S S K], \operatorname{Pr}[V F S \wedge \overline{S S K} \wedge$ $U I K] \leqq \operatorname{Pr}[\overline{S S K} \wedge U I K]$ and $\operatorname{Pr}[\overline{S S K} \wedge \overline{U I K}] \leqq 1$, we have

$\operatorname{Pr}_{L R-I} \leqq \operatorname{Pr}[S S K]+\operatorname{Pr}[\overline{S S K} \wedge U I K]+\operatorname{Pr}[V F S \mid \overline{S S K} \wedge \overline{U I K}]$.

By Lemmas 4, 5 and 6 later, we respectively evaluate three probabilities $\operatorname{Pr}[S S K], \operatorname{Pr}[\overline{S S K} \wedge \overline{U I K}]$ and $\operatorname{Pr}[\overline{S S K} \wedge U I K]$. Thus, we have

$\operatorname{Pr}_{L R-\mathrm{I}} \leqq \operatorname{Pr}[S S K]+\operatorname{Pr}[V F S \mid \overline{S S K} \wedge \overline{U I K}]+\operatorname{Pr}[\overline{S S K} \wedge U I K]$ $\leqq O\left(\left(q^{2} / p\right) 2^{2 \lambda}\right)+O\left(\left(q^{2} / p\right) 2^{\lambda}\right)+O\left(\left(q^{2} / p\right) 2^{2 \lambda}\right)$

$\leqq O\left(\left(q^{2} / p\right) 2^{2 \lambda}\right)$.

Hence, the advantage of $A_{L R-\mathrm{I}}$ breaking the proposed LR-CLS scheme is $O\left(\left(q^{2} / p\right) 2^{2 \lambda}\right)$. By Corollary 1 , if $\lambda<<$ $\frac{\log (p)}{2}$, the proposed LR-CLS scheme is existential unforgeability against adaptive chosen-message attacks.

\section{Lemma 4. $\operatorname{Pr}[S S K] \leqq O\left(\left(q^{2} / p\right) 2^{2 \lambda}\right)$.}

Proof. In the Initial key extract phase of the proposed LR-CLS scheme, a user's initial key is a signature on the user's identity ID generated by Galindo and Vivek's LRS signature scheme in [18]. Therefore, by applying the Lemma 5, we have $\operatorname{Pr}[S S K] \leqq O\left(\left(q^{2} / p\right) 2^{2 \lambda}\right)$. $\square$

Lemma 5. $\operatorname{Pr}[V F S \mid \overline{S S K} \wedge \overline{U I K}] \leqq O\left(\left(q^{2} / p\right) 2^{\curlywedge}\right)$.

Proof. In Theorem 1, we have proved that an adversary has the success probability $O\left(q^{2} / p\right)$ to break the non-leakage version $\Pi_{N L}$ of the proposed LR-CLS scheme. For both events $\overline{S S K}$ and $\overline{U I K}, A_{L R-\mathrm{I}}$ can learn at most $\lambda$ bits information about the user's current private key $\left(D I D_{j, 1}, D I D_{j, 2}\right)$. Therefore, the probability of $A_{L R-I}$ forging a valid signature by using at most $\lambda$ bits leakage information is $\operatorname{Pr}[V F S \mid \overline{S S K} \wedge \overline{U I K}] \leqq$ $O\left(\left(q^{2} / p\right) 2^{\lambda}\right)$.

Lemma 6. $\operatorname{Pr}[\overline{S S K} \wedge U I K] \leqq O\left(\left(q^{2} / p\right) 2^{2 \lambda}\right)$.

Proof. For the event $\overline{S S K}, A_{L R-\mathrm{I}}$ is unable to obtain any useful information by Initial key extract leak query, but may obtain the useful information by Sign leak query to forge a signature. However, $A_{L R-\mathrm{I}}$ may obtain useful information by Sign leak query. In this case, $\operatorname{Pr}[\overline{S S K} \wedge U I K]$ is the event that $A_{L R-\mathrm{I}}$ can obtain the partial information of a user's first initial key by the Sign leak query with two leakage functions $f_{S, i}$ and $h_{S, i}$. Hence, the probability to forge a signature under the event $\overline{S S K} \wedge U I K$ is identical to the probability $\operatorname{Pr}[S S K]$ of generating a user's first initial key under the event $S S K$. Therefore, by Lemma 4, we have $\operatorname{Pr}[\overline{S S K} \wedge U I K] \leqq O\left(\left(q^{2} / p\right) 2^{2 \lambda}\right)$.

Theorem 4. In the generic bilinear group model, the proposed LR-CLS scheme is provably secure against the Type II adversary (honest-but-curious KGC) under the continual leakage model.

Proof: We have proven that the non-leakage version of our proposed scheme is secure against the Type II adversary in Theorem 2. Here, the adversary is allowed to issue an extra query, i.e. Sign leak query. Let $A_{L R-\mathrm{II}}$ be a Type I adversary who can break our LR-CLS scheme $\Pi_{L R}$ while $A_{L R-\text { II }}$ is allowed to issue all the queries at most $q$ times. The advantage of $A_{L R \text {-II }}$ is defined as the probability that $A_{L R-\mathrm{I}}$ wins the following game $g_{L R-\mathrm{II}}$ played with a challenger $C$.

Game $g_{L R-\mathrm{II}}$ In the game $g_{L R-I I}$, there are three phases, namely, Setup, Queries and Forgery phases. At the end of this game, $A_{L R \text {-II }}$ outputs a forgery signature. In Queries phase, $A_{L R-\text { II }}$ may issue seven kinds of queries in any order at most $q$ times. Three phases are described as below:

- Setup phase: This phase is identical to that of the game $g_{N L-I I}$.

_ Queries phase: In addition to the six kinds of queries in the game $g_{N L-I I}, A_{L R-\text { II }}$ may issue one extra leakage query (Sign leak query). In order to represent the leakage information, two leakage functions $f_{S, j}$ and $h_{S, j}$ are used to model the ability of the adversary for Sign-1 and Sign-2 of a user's $j$-th Sign round. Note that two leakage functions $f_{S, j}$ and $h_{S, j}$ respectively generate the leakage information $\Lambda f_{S, j}$ and $\Lambda h_{S, j}$. Meanwhile, two initial-empty lists $L_{f, S}$ and $L_{h, S}$ are used to record the related leakage functions and leakage information:

$L_{f, S}=\left\{\left(f_{S, j}, \Lambda f_{S, j}\right), 1 \leqq j \leqq q_{S}\right\}, L_{h, S}=\left\{\left(h_{S, j}, \Lambda h_{S, j}\right), 1 \leqq j \leqq q_{S}\right\}$.

_ Sign leakquery $\left(f_{S, j}, h_{S, j}, j\right):$ This query is identical to the Sign leak query described in the game $g_{L R-\mathrm{I}}$.

- Forgery phase: In this phase, the type II adversary $A_{L R-\mathrm{II}}$ outputs a forgery signature $\left(m^{*}, I D^{*}, \sigma^{*}=\left(\xi_{G, f, i, 1}\right.\right.$, $\left.\xi_{G, f, 2,2}{ }^{*}\right)$. It is worth mentioning, that there are two restrictions: (1) $I D^{*}$ has never been issued during the Secret key extract query $Q_{S E}$; (2) $\left(m^{*}, I D^{*}\right)$ has never been issued during the Sign query $Q s$. 
By making use of the leakage functions, it is obvious that the success probability (advantage) of $A_{L R-\text { II }}$ in $g_{L R-\text { II }}$ is higher than that of $A_{N L \text {-II }}$ in the game $g_{N L \text {-II }}$. For the Sign leak query with two leakage functions $f_{S, j}$ and $h_{S, j}$, the adversary $A_{L R-\text { II }}$ can obtain partial information of $\left(D I D_{j-1,1}, \eta_{j}, b_{j}, c_{j}\right)$ and $\left(D I D_{j-1,2}, b_{j}, c_{j}\right)$ by the leakage information $\Lambda f_{S, j}$ and $\Lambda h_{S, j}$, respectively. The discussions on the partial leakage information of $\eta_{j}$, $\left(D I D_{j-1,1}\right.$, $\left.D I D_{j-1,2}\right), b_{j}$ and $c_{j}$ are the same with those in Theorem 3. Here, we evaluate the probability that $A_{L R \text {-II }}$ wins the game $g_{L R-\mathrm{II}}$, denoted by $\operatorname{Pr}_{L R-\mathrm{II}}$. Note that since the type II adversary $A_{L R \text {-II }}$ has obtained the system secret key $X$, $A_{L R-\text { II }}$ can generate the user's initial key $D I D_{0}$ of any entity. In such a case, $A_{L R-\text { II }}$ can forge a valid signature if $A_{L R-\text { II }}$ gets the user's secret key SID. In the following, we define two events of $\operatorname{Pr}_{L R-I I}$ : (1) The event USK denotes that $A_{L R-\text { II }}$ can get the user's secret key SID completely by two leakage functions $f_{S, j}$ and $h_{S, j}$. The event $\overline{U S K}$ is the complement event of USK; (2) The event $V F S$ denotes that $A_{L R-\text { II }}$ can generate a valid forgery signature. Therefore, the probability that $A_{L R-\text { II }}$ wins the game $g_{L R-I I}$ is bounded by

$\operatorname{Pr}_{L R-\mathrm{II}}=\operatorname{Pr}[V F S]=\operatorname{Pr}[V F S \wedge U S K]+\operatorname{Pr}[V F S \wedge \overline{U S K}]$ $=\operatorname{Pr}[V F S \wedge U S K]+\operatorname{Pr}[V F S \mid \overline{U S K}] \cdot \operatorname{Pr}[\overline{U S K}]$.

Since $\operatorname{Pr}[V F S \wedge U S K] \leqq \operatorname{Pr}[U S K]$ and $\operatorname{Pr}[\overline{U S K}] \leqq 1$, we have $\operatorname{Pr}_{L R-\mathrm{II}} \leqq \operatorname{Pr}[U S K]+\operatorname{Pr}[V F S \mid \overline{U S K}]$.

By Lemmas 7 and 8 later, we respectively evaluate two probabilities $\operatorname{Pr}[U S K]$ and $\operatorname{Pr}[V F S \mid \overline{U S K}]$. Thus, we have

$\operatorname{Pr}_{L R-\mathrm{II}} \leqq \operatorname{Pr}[U S K]+\operatorname{Pr}[V F S \mid \overline{U S K}]$,

$\leqq O\left((1 / p)^{*} 2^{2 \lambda}\right)+O\left(\left(q^{2} / p\right)^{*} 2^{2 \lambda}\right)$

$\leqq O\left(\left(q^{2} / p\right)^{*} 2^{2 \lambda}\right)$.

Hence, the advantage of $A_{L R \text {-II }}$ breaking the proposed LR-CLS scheme is $O\left(\left(q^{2} / p\right) 2^{2 \lambda}\right)$. By Corollary 1, if $\lambda<<$ $\frac{\log (p)}{2}$, the proposed LR-CLS scheme is existential unforgeability against adaptive chosen-message attacks.

Lemma \%. $\operatorname{Pr}[U S K] \leqq O\left((1 / p)^{*} 2^{2 \lambda}\right)$.

Proof. Since $A_{L R-\text { II }}$ can learn at most $2 \lambda$ bits information for the user current secret key by the Sign leak query with two leakage functions $f_{S, i}$ and $h_{S, i}$, we have $\operatorname{Pr}[U S K] \leqq O\left((1 / p)^{*} 2^{2 \lambda}\right)$.

\section{Lemma 8. $\operatorname{Pr}[V F S \mid \overline{U S K}] \leqq O\left(\left(q^{2} / p\right)^{*} 2^{\lambda}\right)$.}

Proof. In Theorem 3, we have proved that an adversary has the success probability $O\left(q^{2} / p\right)$ to break the non-leakage version $\Pi_{\underline{N L}}$ of the proposed LR-CLS scheme. For the event $\overline{U S K}, A_{L R-\text { II }}$ can learn at most $\lambda$ bits information about the user's current secret key $\left(S I D_{j, 1}, S I D_{j, 2}\right)$. Therefore, the probability of $A_{L R-\text { II }}$ forging a valid signature by using at most $\lambda$ bits leakage information is $\operatorname{Pr}[V F S \mid \overline{U S K}] \leqq O\left(\left(q^{2} / p\right)^{*} 2^{\lambda}\right)$.

\section{Performance Analysis}

Our proposed scheme is the first LR-CLS scheme under the continual leakage model. To achieve leakage-resilient property, we added some extra computations in our scheme so that the performance is not better than that of the previously proposed CLS schemes [19-23, 44, 46]. Fortunately, the performance of our LR-CLS scheme is still suitable for mobile devices. For practicality, a suitable bilinear pairing group to implement our LR-IBS scheme is the pairing-friendly curves presented by Scott in [35]. In the following, we demonstrate the performance analysis of the proposed LR-CLS scheme.

For convenience, we define the following notations to analyze the computational costs.

- $T_{p}$ : The time of executing a bilinear pairing operation $e: G \times G \rightarrow G_{T}$.

- $T_{e}$ : The time of executing an exponentiation operation in $G$.

Here, we analyze the computational costs of the proposed LR-CLS scheme in terms of Initial key extract, Sign and Verify phases. The Initial key extract phase including Extract-1 and Extract-2 sub-algorithms requires $5 T_{e}$ to update the current system secret key to $\left(S_{i 1}, S_{i 2}\right)$ and produce the first initial key of a user with identity ID. The Sign phase including Sign-1 and Sign-2 sub-algorithms requires $7 T_{e}$ to generate a signature $\sigma$ while updating the current private key of the signer with identity ID. In addition, the Verify phase requires $3 T_{p}+2 T_{e}$ to validate a signature.

We use the newest implementation results of the related operations in the generic bilinear group made by Galindo et al. [17] to measure the computational 
cost of the proposed LR-CLS scheme. Their implementation environment is presented as follows. The processor is an ARM Cortex-M3 CPU. The group $G$ is an elliptic curve group over $F_{p}$ with a bit-length of 254 bits while $G_{T}$ is a subgroup of the multiplicative group of the extension field $F_{p 12}$. The required computational costs (in 106 clock cycles) of $T_{e}$ and $T_{p}$ are 4.5 and 65 , respectively. Here, the multiplication operation is ignored as compared with $T_{e}$ and $T_{p}$. Table 1 lists the required computational costs (in 106 clock cycles) of the Initial key extract, Sign and Verify phases in the proposed LR-CLS scheme. It is obvious that the proposed LR-CLS scheme is well suitable for mobile devices.

Table 1

Computational costs of the proposed LR-CLS scheme

\begin{tabular}{c|c|c}
\hline Phase & $\begin{array}{c}\text { Required } \\
\text { operations }\end{array}$ & $\begin{array}{c}\text { Running cost } \\
\text { (in 106 clock cycles) }\end{array}$ \\
\hline $\begin{array}{c}\text { Initial key } \\
\text { extract }\end{array}$ & $5 T_{e}$ & 22.5 \\
\hline Sign & $7 T_{e}$ & 31.5 \\
\hline Verify & $3 T_{p}+2 T_{e}$ & 204 \\
\hline
\end{tabular}

\section{References}

1. Al-Riyami, S. S., Paterson, K. G. Certificateless Public Key Cryptography. In: ASIACRYPT'03, LNCS, 2894, Springer, Belin-Heidelberg, 2003, 452-473. https://doi. org/10.1007/978-3-540-40061-5_29

2. Alwen, J., Dodis, Y., Naor, M., Segev, G., Walfish, S., Wichs, D. Public-Key Encryption in the Bounded-Retrieval Model. In: EUROCRYPT'10, LNCS, 6110, Springer, Belin-Heidelberg, 2010, 113-134. https://doi. org/10.1007/978-3-642-13190-5_6

3. Alwen, J., Dodis, Y., Wichs, D. Leakage-Resilient Public-Key Cryptography in the Bounded-Retrieval Model. In: CRYPTO’09, LNCS, 5677, Springer, Belin-Heidelberg, 2009, 36-54. https://doi.org/10.10077/978-3-64203356-8_3

4. Biham, E., Carmeli, Y., Shamir, A. Bug Attacks. In: CRYPTO’08, LNCS, 5157\%, Springer, Belin-Heidelberg,

\section{Conclusions}

In this article, we proposed the first LR-CLS scheme under the continual leakage model. We defined the new security notions for LR-CLS schemes under the continual leakage model. In the security notions, there are two kinds of attackers, namely, Type I adversary (outsider) and Type II adversary (honest-but-curious KGC). Both kinds of attackers are extended from the security notions of traditional certificateless signature (CLS) schemes by adding the Initial key extract leak query and the Sign leak query. Type I adversary may obtain not only the leakage information of a user's initial key of the private key in the Sign phase, but also the leakage information of the KGC's system secret key in the Initial key extract phase. Type II adversary knows the initial key of any entity while obtaining the leakage information of a user's secret key of the private key in the Sign phase. In the generic bilinear group model, we demonstrate that our LR-CLS scheme possesses existential unforgeability against adaptive chosen-message attacks for both Type I and Type II adversaries under the continual leakage model.

\section{Acknowledgment}

This research was partially supported by Ministry of Science and Technology, Taiwan, under contract no. MOST105-2221-E-018-013.

2008, 221-240. https://doi.org/10.1007/978-3-54085174-5_13

5. Boneh, D., Boyen, X., Goh, E. J. Hierarchical Identity-Based Encryption with Constant Size Ciphertext. In: EUROCRYPT'05, LNCS, 3494, Springer, Belin-Heidelberg, 2005, 440-456. https://doi. org/10.1007/11426639_26

6. Boneh, D., Demillo, R. A., Lipton, R. J. On the Importance of Checking Cryptographic Protocols for Faults. In: EUROCRYPT'97, LNCS, 1233, Springer, Belin-Heidelberg, 1997, 37-51. https://doi.org/10.1007/3-540-69053-0_4

7. Boneh, D., Franklin, M. Identity-Based Encryption from the Weil Pairing. In: CRYPTO'01, LNCS, 2139, Springer, Belin-Heidelberg, 2001, 213-229. https://doi. org/10.1007/3-540-44647-8_13

8. Boneh, D., Lynn, B., Shacham, H. Short signatures from 
the Weil pairing. In: ASIACRYPT'01, LNCS, 2248, Springer, Belin-Heidelberg, 2001, 514-532. https://doi. org/10.1007/3-540-45682-1_30

9. Brakerski, Z., Kalai, Y. T., Katz, J., Vaikuntanathan, V. Cryptography Resilient to Continual Memory Leakage. Proceedings of 51st Annual IEEE Symposium on Foundations of Computer Science, 2010, 501-510.

10. Brumley, D., Boneh, D. Remote Timing Attacks are Practical. Computer Networks, 2005, 48(5), 701-r16. https://doi.org/10.1016/j.comnet.2005.01.010

11. Dodis, Y., Haralambiev, K., Lopez-Alt, A., Wichs, D. Efficient Public-Key Cryptography in the Presence of Key Leakage. In: ASIACRYPT'10, LNCS, 6477, Springer, Belin-Heidelberg, 2010, 613-631. https://doi. org/10.1007/978-3-642-17373-8_35

12. Dodis, Y., Haralambiev, K., Lopez-Alt, A., Wichs, D. Cryptography Against Continuous Memory Attacks. Proceedings of 51st Annual IEEE Symposium on Foundations of Computer Science, 2010, 511-520. https:// doi.org/10.1109/FOCS.2010.56

13. Dodis, Y., Ostrovsky, R., Reyzin, L., Smith, A. Fuzzy Extractors: How to Generate Strong Keys from Biometrics and Other Noisy Data. SIAM Journal on Computing, 2008, 38(1), 97-139. https://doi.org/10.1137/060651380

14. ElGamal, T. A Public Key Cryptosystem and a Signature Scheme Based on Discrete Logarithms. IEEE Transactions on Information Theory, 1985, 31(4), 469-472. https://doi.org/10.1109/TIT.1985.1057074

15. Faust, S., Hazay, C., Nielsen, J. B., Nordholt, P. S., Zottarel, A. Signature Schemes Secure Against Hardto-Invert Leakage. In: ASIACRYPT'12, LNCS, 7658, Springer, Belin-Heidelberg, 2012, 98-115. https://doi. org/10.1007/978-3-642-34961-4_8

16. Faust, S., Kiltz, E., Pietrzak, K., Rothblum, G. N. Leakage-Resilient Signatures. In: TCC'10, LNCS, 5978, Springer, Belin-Heidelberg, 2010, 343-360. https://doi. org/10.1007/978-3-642-11799-2_21

17. Galindo, D., Grobschadl, J., Liu, Z., Vadnala, P. K., Vivek, S. Implementation of a Leakage-Resilient Elgamal Key Encapsulation Mechanism. Journal of Cryptographic Engineering, 2016, 6(3), 229-238. https://doi. org/10.1007/s13389-016-0121-x

18. Galindo, D., Virek, S. A Practical Leakage-Resilient Signature Scheme in the Generic Group Model. In: SAC'12, LNCS, 7707, Springer, Belin-Heidelberg, 2013, 50-65. https://doi.org/10.100'7/978-3-642-35999-6_4

19. Hu, B., Wong, D., Zhang, Z., Deng, X. Key Replacement Attack Against a Generic Construction of Certificate- less Signature. In: ACISP'06, LNCS, 4058, Springer, Belin-Heidelberg, 2006, 235-346. https://doi. org/10.1007/11780656_20

20. Hu, B., Wong, D., Zhang, Z., Deng, X. Certificateless Signature: A New Security Model and an Improved Generic Construction. Designs, Codes and Cryptography, 2007, 42(2), 109-126. https://doi.org/10.1007/s10623006-9022-9

21. Huang, X., Mu, Y., Susilo, W., Wong, D., Wu, W. Certificateless Signature Revisited. In: ACISP'07, LNCS, 4586, Springer, Belin-Heidelberg, 2007, 308-322. https://doi. org/10.1007/978-3-540-73458-1_23

22. Huang, X., Susilo, W., Mu, Y., Zhang, F. On the Security of Certificateless Signature Schemes from Asiacrypt 2003. In: CANS'05, LNCS, 3810, Springer, Belin-Heidelberg, 2005, 13-25. https://doi.org/10.1007/11599371_2

23. Hung, Y. H., Tseng, Y. M., Huang, S. S. A Revocable Certificateless Short Signature Scheme and Its Authentication Application. Informatica, 2016, 27(3), 549-572. https://doi.org/10.15388/Informatica.2016.99

24. Hwang, Y. H., Liu, J. K., Chow, S. S. M. Certificateless Public Key Encryption Secure Against Malicious KGC Attacks in the Standard Model. Journal of Universal Computer Science, 2008, 14(3), 463-480.

25. Katz, J., Vaikuntanathan, V. Signature Schemes with Bounded Leakage Resilience. In: ASIACRYPT'09, LNCS, 5912, Springer, Belin-Heidelberg, 2009, 703720. https://doi.org/10.1007/978-3-642-10366-7_41

26. Kiltz, E., Pietrzak, K. Leakage Resilient Elgamal Encryption. In: ASIACRYPT'10, LNCS, 6477, Springer, Belin-Heidelberg, 2010, 595-612. https://doi. org/10.1007/978-3-642-17373-8_34

27. Kocher, P., Jaffe, J., Jun, B. Differential Power Analysis. In: CRYPTO'99, LNCS, 1666, Springer, Belin-Heidelberg, 1999, 388-397. https://doi.org/10.1007/3-54048405-1_25

28. Kocher, P. C. Timing Attacks on Implementations of Diffie-Hellman, RSA, DSS, and Other Systems. In: CRYPTO’96, LNCS, 1163, Springer, Belin-Heidelberg, 1996, 104-113. https://doi.org/10.1007/3-540-68697-5_9

29. Libert, B., Quisquater, J. J. On Constructing Certificateless Cryptosystems from Identity Based Encryption. In: PKC'06, LNCS, 3958, Springer, Belin-Heidelberg, 2006, 474-490. https://doi.org/10.1007/11745853_31

30. Lin, H. Y. Secure Certificateless Two-Party Key Agreement with Short Message. Information Technology and Control, 2016, 45(1), 71-76. https://doi.org/10.5755/j01. itc.45.1.12595 
31. Maurer, U., Wolf, S. Lower Bounds on Generic Algorithms in Groups. In: EUROCRYPT'98, LNCS, 1403, Springer, Belin-Heidelberg, 1998, 72-84. https://doi. org/10.1007/BFb0054118

32. Naor, M., Segev, G. Public-Key Cryptosystems Resilient to Key Leakage. In: CRYPTO’09, LNCS, 5677, Springer, Belin-Heidelberg, 2009, 18-35. https://doi. org/10.1007/978-3-642-03356-8_2

33. Rivest, R. L., Shamir, A., Adleman, L. A Method for Obtaining Digital Signatures and Public-Key Cryptosystems. Communications of ACM, 1978, 21(2), 120-126. https://doi.org/10.1145/359340.359342

34. Schwartz, J.T.FastProbabilistic Algorithms forVerification of Polynomial Identities. Journal of the ACM, 1980, 27(4), 701-717. https://doi.org/10.1145/322217.322225

35. Scott, M. On the Efficient Implementation of Pairing-Based Protocols. In: Cryptography and Coding, LNCS, 7089, Springer, Belin-Heidelberg, 2011, 296308. https://doi.org/10.1007/978-3-642-25516-8_18

36. Shamir, A. Identity-Based Cryptosystems and Signature Schemes. In: CRYPTO'84, LNCS, 196, Springer, Belin-Heidelberg, 1984, 47-53.

37. Shoup, V. Lower Bounds for Discrete Logarithms and Related Problems. In: EUROCRYPT'97, LNCS, 1233, Springer, Belin-Heidelberg, 1997, 256-266. https://doi. org/10.1007/3-540-69053-0_18

38. Tang, F., Li, H., Niu, Q., Liang, B. Efficient Leakage-Resilient Signature Schemes in the Generic Bilinear Group Model. In: Information Security Practice and Experience, LNCS, 8434, Springer, Belin-Heidelberg, 2014, 418-432. https://doi.org/10.1007/978-3-31906320-1_31

39. Tsai, T. T., Tseng, Y. M. Revocable Certificateless Public Key Encryption. IEEE Systems Journal, 2015, 9(3), 824-833. https://doi.org/10.1109/JSYST.2013.2289271

40. Tsai, T. T., Tseng, Y. M., Huang, S. S. Efficient Revocable Certificateless Public Key Encryption with a Dele- gated Revocation Authority. Security and Communication Networks, 2015, 8(18), 3713-3725. https://doi. org/10.1002/sec.1294

41. Waters, B. Efficient Identity-Based Encryption Without Random Oracles. In: EUROCRYPT'05, LNCS, 3494, Springer, Belin-Heidelberg, 2005, 114-127. https://doi. org/10.1007/11426639_7

42. Wu, J. D., Tseng, Y. M., Huang, S. S. Leakage-Resilient ID-Based Signature Scheme in the Generic Bilinear Group Model. Security and Communication Networks, 2016, 9(17), 3987-4001. https://doi.org/10.1002/ sec.1580

43. Xiong, H., Yuen, T. H., Zhang, C., Yiu, S. M., He, Y. J. Leakage-Resilient Certificateless Public Key Encryption. Proceedings of the 1st ACM Workshop on Asia Public-Key Cryptography, 2013, 13-22. https://doi. org/10.1145/2484389.2484394

44. Yu, Y., Mu, Y., Wang, G., Xia, Q., Yang, B. Improved Certificateless Signature Scheme Provably Secure in the Standard Model. IET Information Security, 2012, 6(2), 102-110. https://doi.org/10.1049/iet-ifs.2011.0004

45. Yuen, T. H., Chow, S. S. M., Zhang, Y., Yiu, S. M. Identity-Based Encryption Resilient to Continual Auxiliary Leakage. In: EUROCRYPT'12, LNCS, 7237, Springer, Belin-Heidelberg, 2012, 117-134. https://doi. org/10.1007/978-3-642-29011-4_9

46. Yum, D. H., Lee, P. J. Generic Construction of Certificateless Encryption. In: ICCSA'04, LNCS, 3043, Springer, Belin-Heidelberg, 2004, 802-811. https://doi. org/10.1007/978-3-540-24707-4_93

47. Zhou, Y., Yang, B., Zhang, W. Provably Secure and Efficient Leakage-Resilient Certificateless Signcryption Scheme Without Bilinear Pairing. Discrete Applied Mathematics, 2016, 204, 185-202. https://doi. org/10.1016/j.dam.2015.10.018

48. Zippel, R. Probabilistic Algorithms for Sparse Polynomials. In: EUROSAM'79, LNCS, 72, Springer, Belin-Heidelberg, 1979, 216-226. https://doi.org/10.10077/3-54009519-5_73 